\title{
SARS-CoV-2 infection of human ACE2-transgenic mice causes severe lung inflammation and impaired function
}

\author{
Emma S. Winkler 1,2,8, Adam L. Bailey ${ }^{2,8}$, Natasha M. Kafai 1,2, Sharmila Nair', Broc T. McCune', \\ Jinsheng Yu $\mathbb{1}^{3}$, Julie M. Fox ${ }^{1}{ }^{1}$, Rita E. Chen ${ }^{1,2}$, James T. Earnest', Shamus P. Keeler ${ }^{1}{ }^{1,4}$, Jon H. Ritter ${ }^{2}$, \\ Liang-I Kang ${ }^{2}$, Sarah Dort ${ }^{5}$, Annette Robichaud ${ }^{5}$, Richard Head ${ }^{3}$, Michael J. Holtzman ${ }^{10,4}$ and \\ Michael S. Diamond (10) 1,2,6,7凶
}

\begin{abstract}
Although animal models have been evaluated for severe acute respiratory syndrome coronavirus 2 (SARS-CoV-2) infection, none have fully recapitulated the lung disease phenotypes seen in humans who have been hospitalized. Here, we evaluate transgenic mice expressing the human angiotensin I-converting enzyme 2 (ACE2) receptor driven by the cytokeratin-18 (K18) gene promoter (K18-hACE2) as a model of SARS-CoV-2 infection. Intranasal inoculation of SARS-CoV-2 in K18-hACE2 mice results in high levels of viral infection in lungs, with spread to other organs. A decline in pulmonary function occurs 4 days after peak viral titer and correlates with infiltration of monocytes, neutrophils and activated T cells. SARS-CoV-2-infected lung tissues show a massively upregulated innate immune response with signatures of nuclear factor-kB-dependent, type I and II interferon signaling, and leukocyte activation pathways. Thus, the K18-hACE2 model of SARS-CoV-2 infection shares many features of severe COVID-19 infection and can be used to define the basis of lung disease and test immune and antiviral-based countermeasures.
\end{abstract}

S evere acute respiratory syndrome coronavirus 2 (SARS-CoV-2) is the recently emerged RNA virus responsible for the coronavirus disease 2019 (COVID-19) pandemic. Clinical disease is variable, ranging from asymptomatic infection to multi-organ failure and death, with a case fatality rate of $\sim 5 \%$. Binding of the SARS-CoV-2 spike protein to human angiotensin I-converting enzyme 2 (hACE2) primarily targets the virus to type II pneumocytes within the lung, resulting in injury, inflammation and subsequent respiratory distress ${ }^{1,2}$. Other COVID-19 manifestations (for example, cardiac dysfunction, coagulopathy and gastrointestinal tract symptoms) suggest that extra-pulmonary sites of infection contribute to disease pathogenesis in some patients ${ }^{3}$.

The development of countermeasures that reduce COVID-19 morbidity and mortality is a priority for the global research community, and animal models are essential for this effort. Although several animal species used in laboratory research have been evaluated for susceptibility to SARS-CoV-2 infection, none have recapitulated the severe disease seen in humans who have been hospitalized. Hamsters, ferrets and non-human primates develop mild to moderate viral disease and recover spontaneously ${ }^{4,5}$. Conventional laboratory strains of mice cannot be infected efficiently with SARS-CoV-2 because hACE2, but not mouse ACE2, supports SARS-CoV-2 binding $^{6,7}$. Multiple strategies for introducing hACE2 into mice have been developed including: (1) transient introduction of hACE2 via adenoviral or adenoviral-associated vectors ${ }^{8,9} ;(2)$ expression of
hACE2 as a transgene driven by heterologous gene promoters ${ }^{10,11}$; or (3) expression of hACE2 by the mouse ACE2 promoter $^{12,13}$. While these animals all support SARS-CoV-2 infection, only the model with hACE2 expression driven by the HFH4 promoter $^{11}$ develops severe disease. Thus, additional animal models are needed to enable understanding of the biology of severe SARS-CoV-2 infection in the lung and evaluation of the efficacy of antiviral and immunomodulatory countermeasures.

K18-hACE2-transgenic mice, in which hACE2 expression is driven by the epithelial cell cytokeratin-18 (K18) promoter $^{14}$, were originally developed for the study of SARS-CoV pathogenesis and cause lethal infection ${ }^{10}$. Here, we evaluate heterozygous hACE2-transgenic mice as a model for severe COVID-19 disease. After intranasal SARS-CoV-2 inoculation, K18-hACE2 mice rapidly lost weight starting at $4 \mathrm{~d}$ post-infection (dpi) and began to succumb to disease at $7 \mathrm{dpi}$. High levels of viral RNA and infectious virus were detected in the lungs of infected animals at 2, 4 and $7 \mathrm{dpi}$ by quantitative PCR with reverse transcription (RT-qPCR), in situ hybridization and plaque-forming assays. Infection was accompanied by declines in multiple parameters of pulmonary function, substantial cellular infiltrates in the lung (composed of monocytes, neutrophils and activated T cells), high levels of pro-inflammatory cytokines and chemokines in lung homogenates, and severe interstitial and consolidative pneumonia. Because of its severe disease and intense immune cell infiltration, the K18-hACE2 model of

\footnotetext{
'Department of Medicine, Washington University School of Medicine, St. Louis, MO, USA. ${ }^{2}$ Department of Pathology and Immunology, Washington University School of Medicine, St. Louis, MO, USA. ${ }^{3}$ Department of Genetics, Washington University School of Medicine, St. Louis, MO, USA. ${ }^{4}$ Division of Pulmonary and Critical Care Medicine, Washington University School of Medicine, St. Louis, MO, USA. ${ }^{5}$ SCIREQ Scientific Respiratory Equipment, Montreal, Quebec, Canada. ${ }^{6}$ Department of Molecular Microbiology, Washington University School of Medicine, St. Louis, MO, USA. ${ }^{7}$ The Andrew M. and Jane M. Bursky Center for Human Immunology and Immunotherapy Programs, Washington University School of Medicine, St. Louis, MO, USA. ${ }^{8}$ These authors contributed equally: Emma S. Winkler, Adam L. Bailey.凶e-mail: diamond@wusm.wustl.edu
} 
a

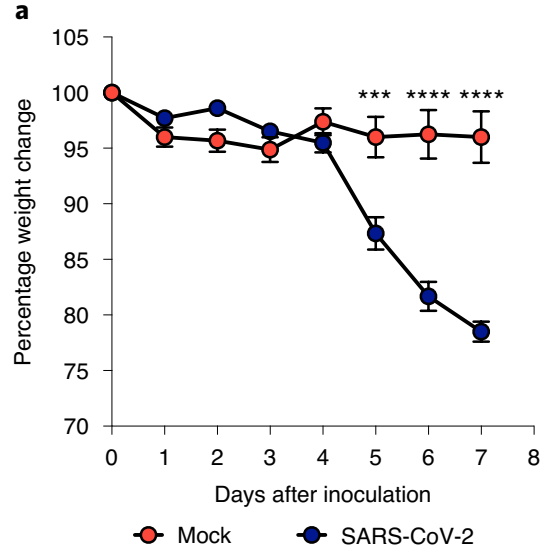

d

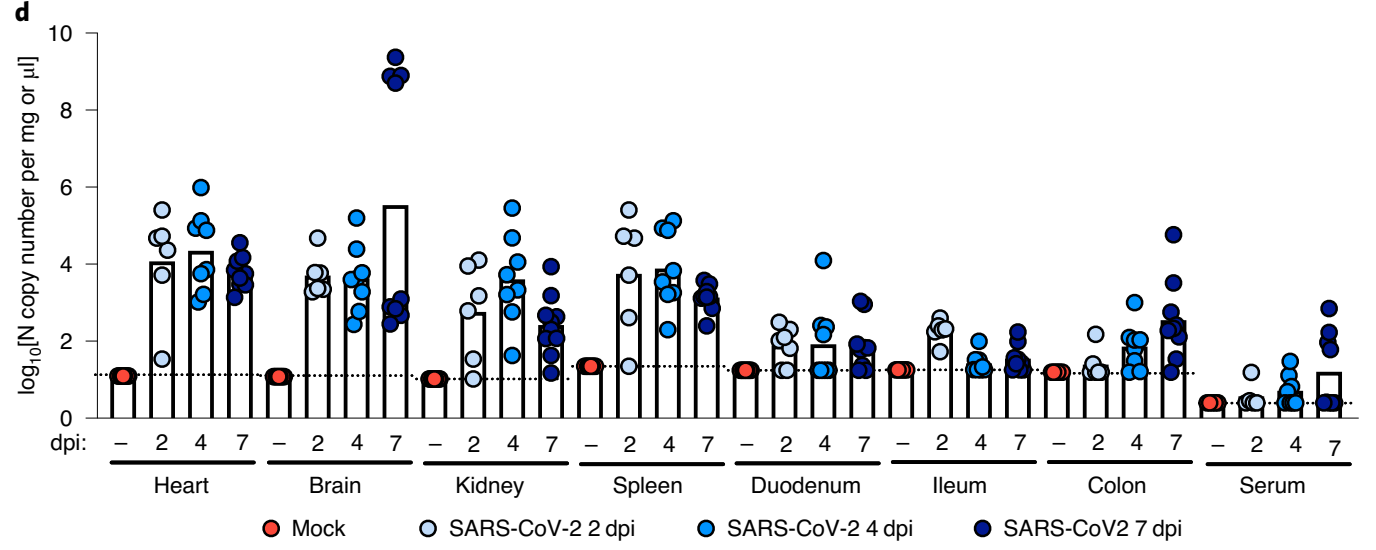

b

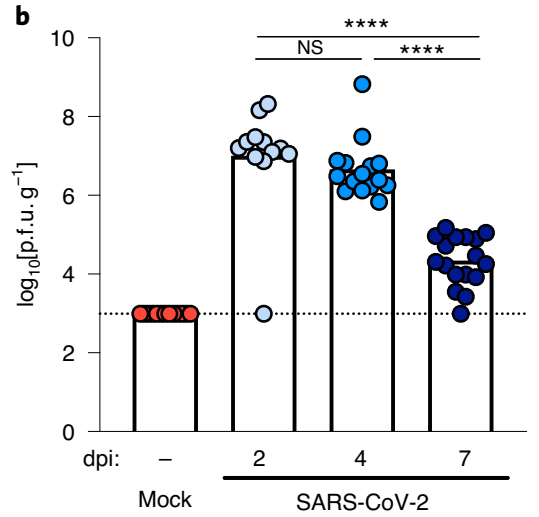

c

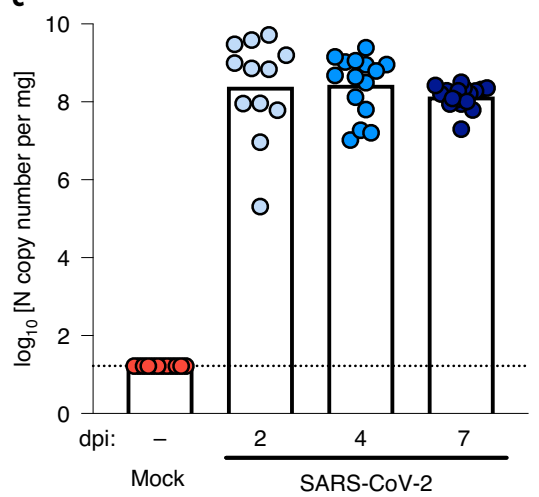

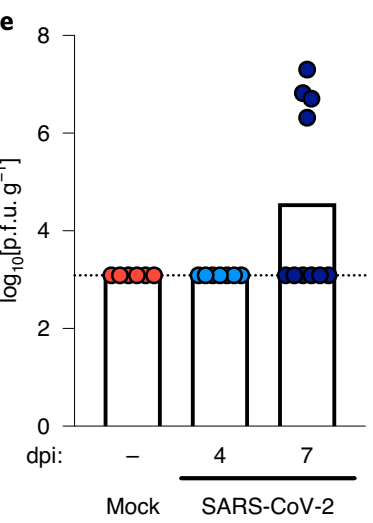

Fig. 1 | SARS-CoV-2 infection in K18-hACE2 mice. a-e, Eight- to nine-week-old male and female K18-hACE2-transgenic mice were inoculated via the intranasal route with $2.5 \times 10^{4}$ p.f.u. SARS-CoV-2. a, Weight change was monitored (two experiments; $n=10 ;$ two-way ANOVA: $\star \star \star P=0.0002 ;$ $\star \star \star \star P<0.0001$; symbols represent means \pm s.e.m.). b,c, Viral burden in the lungs was analyzed at 2, 4 and 7 dpi by plaque assay for infectious virus (b) and RT-qPCR for viral RNA levels (c). For b, one-way ANOVA with Dunnett's test was used to determine significance ( ${ }^{\star \star \star} P<0.0001$; NS, not significant). d, Viral RNA levels in the indicated tissues (heart, brain, kidney, spleen, serum and gastrointestinal tract) at 2, 4 and $7 \mathrm{dpi}$, as measured by RT-qPCR. e, Viral burden in the brains, as measured by plaque assay. For b-e, two experiments were performed $(n=10)$. Bars represent means and dotted horizontal lines indicate the limit of detection.

SARS-CoV-2 infection may facilitate evaluation of immunomodulatory and antiviral drugs against COVID-19 and our understanding of immune-mediated mechanisms of pathogenesis.

\section{Results}

K18-hACE2 mice are highly susceptible to SARS-CoV-2 infection. We inoculated 8-week-old heterozygous K18-hACE2 mice of both sexes via the intranasal route with $2.5 \times 10^{4}$ plaque-forming units (p.f.u.) of SARS-CoV-2 (strain 2019n-CoV/USA_WA1/2020). Beginning at $4 \mathrm{dpi}, \mathrm{K} 18$-hACE2 mice demonstrated marked weight loss, and by $7 \mathrm{dpi}$ most animals had lost approximately $25 \%$ of their body weight (Fig. 1a), with many becoming moribund. No sex-based differences in disease were observed. Intranasal inoculation with a lower $\left(1 \times 10^{2}\right.$ p.f.u. $)$ dose also resulted in substantial weight loss by $6 \mathrm{dpi}$, although this was delayed compared with a higher virus inoculum (Extended Data Fig. 1a). High levels of infectious SARS-CoV-2 (Fig. 1b) and viral RNA (Fig. 1c) were detected in lung homogenates at 2, 4 and $7 \mathrm{dpi}$, whereas lower levels were present in other tissues (for example, the heart, spleen and kidney). Virtually no viral RNA was measured in gastrointestinal tract tissues or in circulation until $7 \mathrm{dpi}$ in the serum and colon, and this was only in a subset of animals (Fig. 1d). The tissues supporting SARS-CoV-2 infection in this model mirrored the pattern of hACE2 expression, with the highest receptor levels in the lungs, colon, kidney and brain (Extended Data Fig. 1b). Levels of hACE2 declined in the lung over the course of infection (Extended Data Fig. 1c), suggesting either receptor downregulation, hACE2 shedding or death of hACE2-expressing cells after SARS-CoV-2 infection. A subset of infected K18-hACE2 mice had high levels of viral RNA and infectious virus in the brain (Fig. 1d,e), consistent with previous reports on SARS-CoV and SARS-CoV-2 in hACE2-transgenic mice ${ }^{10,13,15}$. As no infectious virus and only low levels of viral RNA were detected in the brain of the majority $(60 \%)$ of animals, the observed clinical disease is more consistent with lung and not brain infection. Staining for viral RNA in brain tissue by in situ hybridization showed that only one of six animals was positive at $7 \mathrm{dpi}$.; this animal had disseminated infection throughout the cerebral cortex with noticeable sparing of the olfactory bulb and cerebellum (Extended Data Fig. 2a).

Histopathological changes in the lung after SARS-CoV-2 infection. Analysis of hematoxylin and eosin-stained lung sections from K18-hACE2 mice infected with SARS-CoV-2 (Fig. 2a) showed a progressive inflammatory process. At $2 \mathrm{dpi}$, we observed accumulation of immune cells confined predominantly to perivascular sites. By $4 \mathrm{dpi}$, these immune cell infiltrates involved a greater area of the lung with focal collections into adjacent alveolar spaces with alveolar wall thickening. By $7 \mathrm{dpi}$, immune cells, including neutrophils and mononuclear cells, were found throughout the lung in alveolar and interstitial locations, along with interstitial edema and consolidation. To correlate histopathological findings with sites 

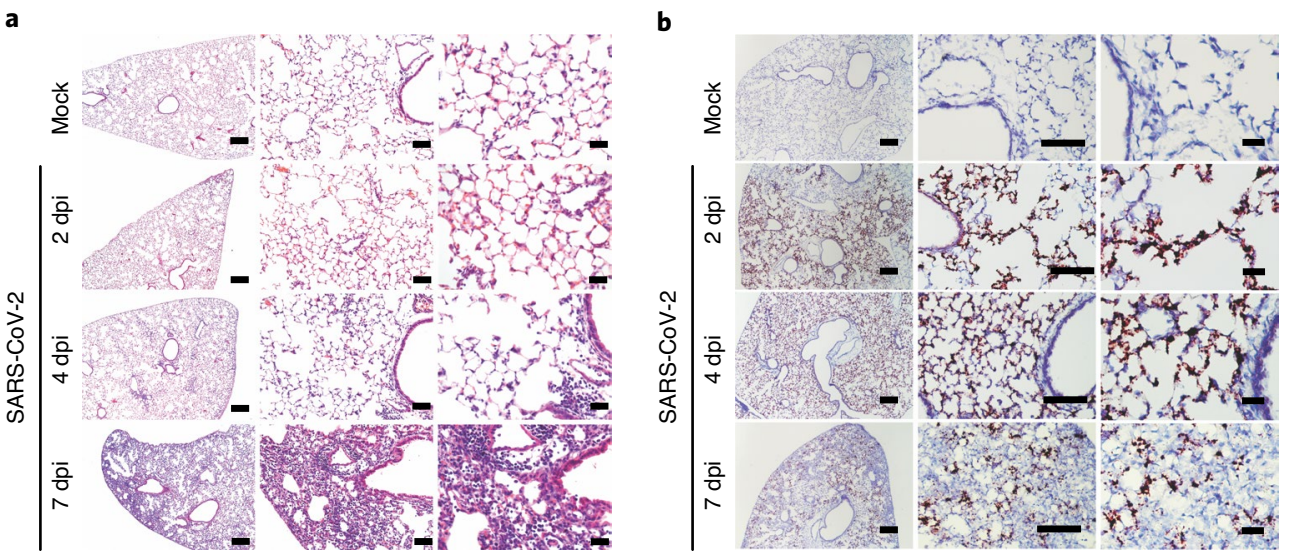

Fig. 2 | Histopathological analysis of SARS-CoV-2 infection in K18-hACE2 mice. a, Hematoxylin and eosin staining of lung sections from K18-hACE2 mice following mock infection or after intranasal infection with $2.5 \times 10^{4}$ p.f.u. SARS-CoV-2 at 2, 4 and 7 dpi. Images show low- (left; scale bars, $250 \mu$ m), medium- (middle; scale bars, $50 \mu \mathrm{m}$ ) and high-power magnification (right; scale bars, $25 \mu \mathrm{m}$ ). b, SARS-CoV-2 RNA in situ hybridization of lung sections from K18-hACE2 mice for the conditions described in a. Images show low- (left; scale bars, $100 \mu \mathrm{m}$ ), medium- (middle; scale bars, $100 \mu \mathrm{m}$ ) and high-power magnification (right; scale bars, $10 \mu \mathrm{m}$ ). In $\mathbf{a}$ and $\mathbf{b}$, images are representative of $n=6$ per group.

of SARS-CoV-2 infection, we also stained lung sections for viral RNA using in situ hybridization (Fig. 2b). At 2 dpi, expression of SARS-CoV-2 RNA was localized predominantly to alveolar epithelial cells and a few airway epithelial cells. This pattern also was seen at $4 \mathrm{dpi}$, but with more diffuse spread throughout the lung. By $7 \mathrm{dpi}$, the level of viral RNA expression was diminished and associated with cellular debris and collapsed alveoli. No significant viral RNA signal was localized to immune cells. Together, these findings provide evidence of a progressive and widespread viral pneumonia with perivascular and pan-alveolar inflammation characterized by immune cell infiltration, edema and lung consolidation.

Extra-pulmonary histopathology after SARS-CoV-2 infection. We examined additional tissues implicated in the pathogenesis of severe COVID-19 in humans, including the brain, heart, liver, kidney and spleen. Brain tissues of K18-hACE2 mice with minimal or no detectable SARS-CoV-2 infection appeared normal without evidence of microglial activation or inflammation, whereas the one brain recovered with a high level of infection at $7 \mathrm{dpi}$ showed inflamed meningeal vessels with extravasation of immune cells (for example, neutrophils, lymphocytes and monocytes) into the brain parenchyma, inflammation of the subarachnoid space and underlying parenchyma including microglial activation (Extended Data Fig. 2b). Abnormalities were observed in two out of nine hearts at $4 \mathrm{dpi}$ (for example, scattered hypereosinophilic cardiomyocytes with pyknotic nuclei) and most livers at 4 and $7 \mathrm{dpi}$ (for example, areas of inflammatory cell infiltrates and hepatocyte loss) (Extended Data Fig. 2c,d). In one kidney at $4 \mathrm{dpi}$, we observed focal acute tubular injury (Extended Data Fig. 2e); otherwise, the kidneys showed no apparent abnormalities. The spleen in SARS-CoV-2-infected K18-hACE2 mice appeared normal (Extended Data Fig. 2f), and fibrin thrombi were not detected in any of the extra-pulmonary organs examined.

Pathophysiology of SARS-CoV-2 infection. To assess for clinically relevant changes in physiology over the course of SARS-CoV-2 infection in K18-hACE2 mice, we measured clinical chemistry and hematological parameters from peripheral blood samples (Fig. 3). Plasma levels of sodium, potassium and chloride concentrations and the anion gap all trended slightly downward at 7 dpi (Fig. 3a-d), whereas plasma bicarbonate noticeably increased (Fig. 3e), possibly as a result of poor gas exchange resulting from lung pathology or decreased respiratory drive. Other plasma analytes, including calcium, glucose and blood urea nitrogen, were unchanged (Fig. $3 \mathrm{f}-\mathrm{h}$ ). Hematocrit and plasma hemoglobin levels increased later in the course of infection, possibly because of reduced water intake and hemoconcentration (Fig. 3i,j). We also observed a modest prolongation in the prothrombin time at $7 \mathrm{dpi}$ that was preceded by an increase in D-dimer concentrations on 2 and 4 dpi (Fig. 3k,1).

Next, we examined the impact of SARS-CoV-2 infection on pulmonary and cardiac function using a treadmill stress test to assess exercise tolerance (Fig. 3m). Compared with mock-infected controls, at $4 \mathrm{dpi}$, a subset of SARS-CoV-2-infected K18-hACE2 mice began to show reduced exercise tolerance, as measured by decreased distance traveled. However, by $5 \mathrm{dpi}$, all infected K18-hACE2 mice had substantially reduced exercise tolerance compared with mock-infected animals or their own pre-infection baseline performance (Fig. $3 \mathrm{~m}$ ).

To examine changes to the biophysical properties of the lung over the course of SARS-CoV-2 infection, we mechanically ventilated mice via tracheostomy and performed several forced oscillation tests to determine various respiratory mechanics parameters (Fig. $3 n-w)$. Infected animals showed normal lung biomechanics at 2 and 4 dpi but had markedly abnormal values in most parameters at $7 \mathrm{dpi}$ relative to mock-infected controls. These abnormalities included reduced inspiratory capacity as well as increased respiratory system resistance and elastance (Fig. $3 n-p)$. Collectively, these changes resulted in a downward deflection of the pressure-volume loop with concomitant decreases in static compliance (Fig. 3q,r), the shape-describing $K$ parameter (representing the curvature of the upper portion of the deflation limb of the pressure-volume curve) (Fig. 3s) and loop hysteresis (Fig. 3t), which together indicated reduced lung compliance and distensibility. Further analysis using broadband forced oscillation maneuvers ${ }^{16}$ revealed that SARS-CoV2 -infected mice at $7 \mathrm{dpi}$ had relatively normal Newtonian resistance (Fig. $3 \mathrm{u}$ ), which is primarily a reflection of resistance in larger conducting airways. In contrast, mice at $7 \mathrm{dpi}$ had marked increases in tissue damping (Fig. 3v) and elastance (Fig. 3w); these parameters measure the dissipation and storage of oscillatory energy in parenchymal tissue and reflect tissue and peripheral airway resistance and elastic recoil (that is, tissue stiffness), respectively. The measurements of mechanical properties of the respiratory system suggest that SARS-CoV-2 infection in K18-hACE2 mice predominantly causes disease in the alveoli and lung parenchyma, and not in the 
a

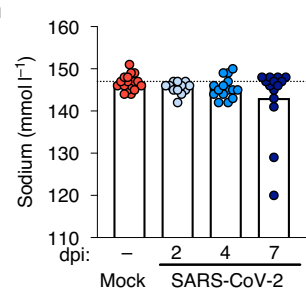

f
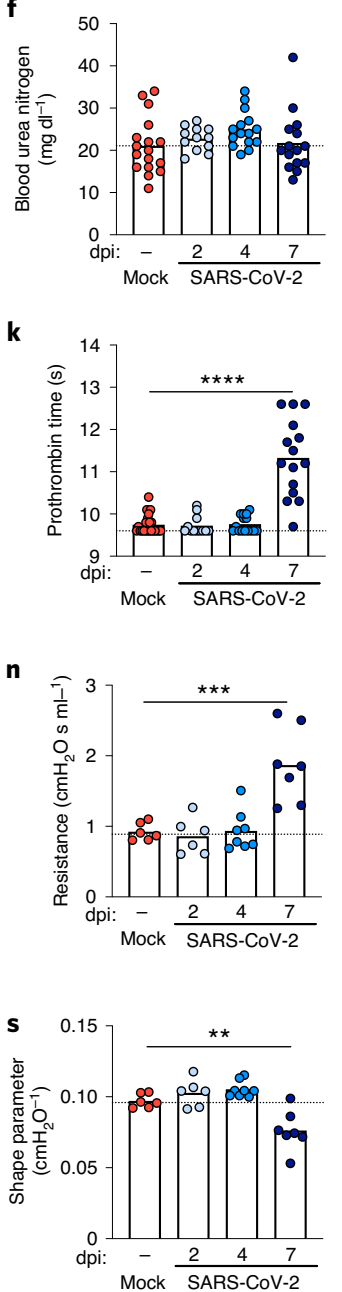

b

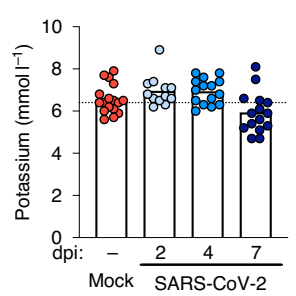

g

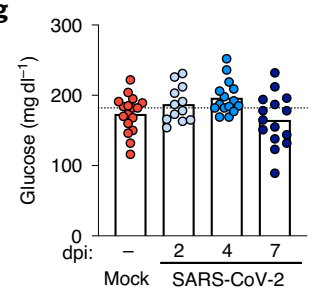

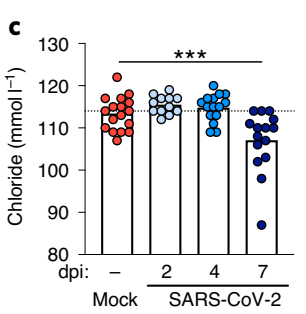
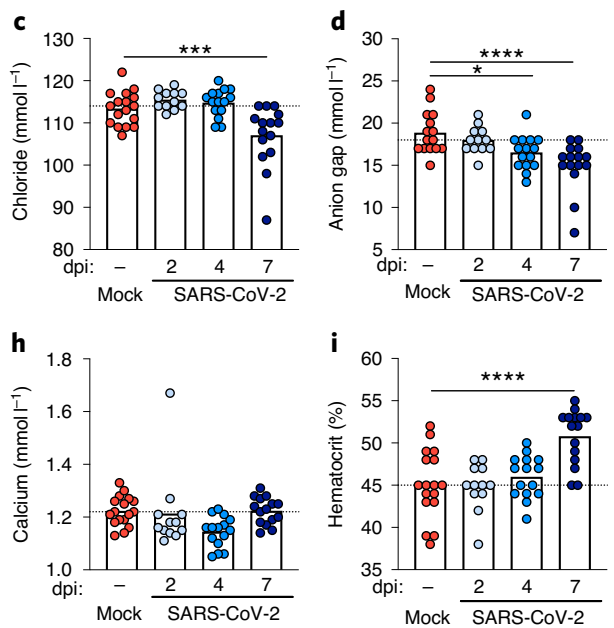
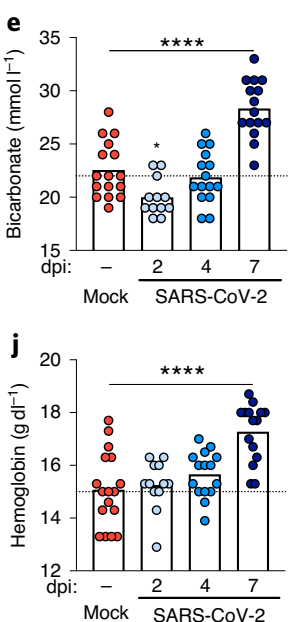
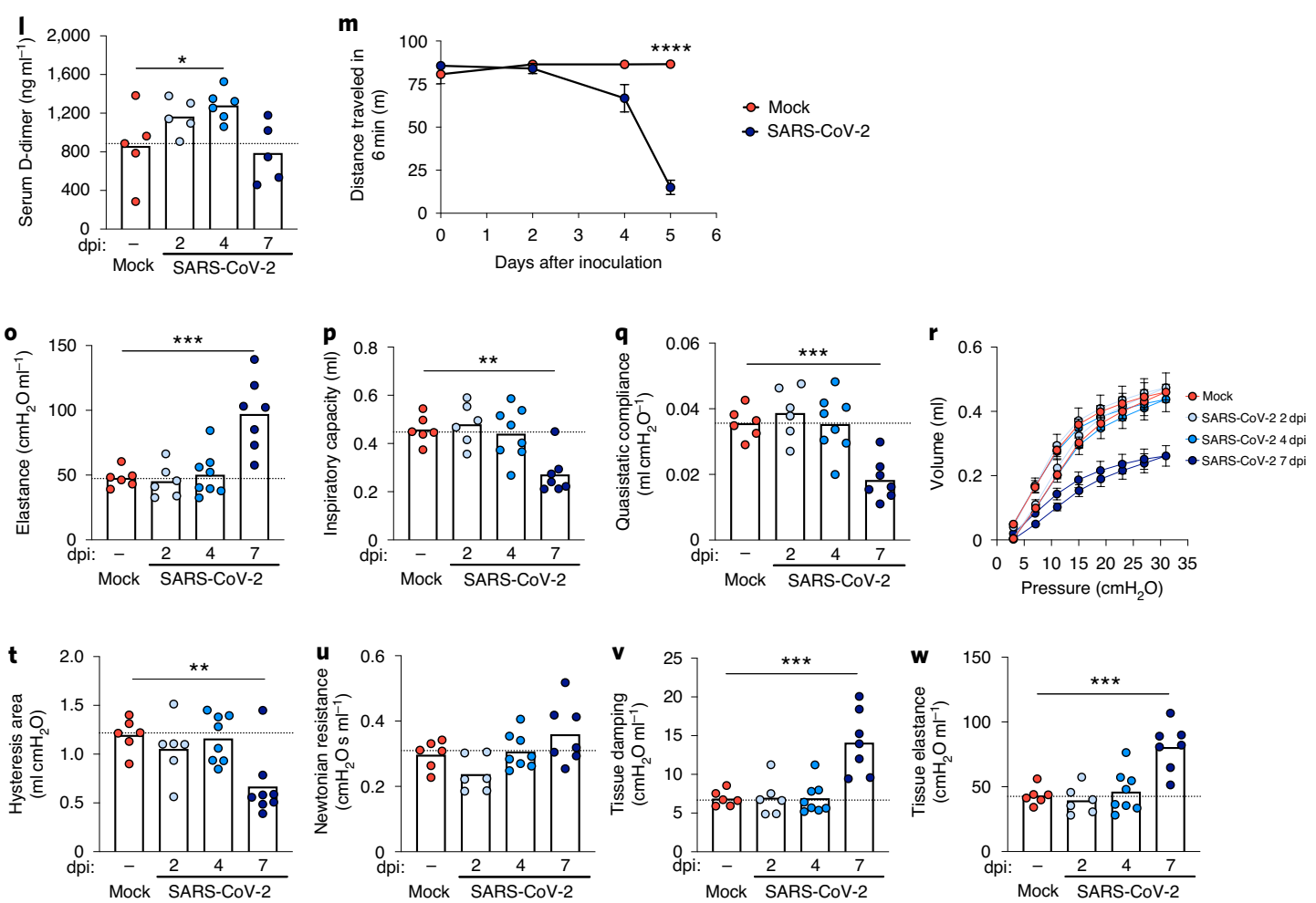

Fig. 3 | Functional consequences of SARS-CoV-2 infection in K18-hACE2 mice. a-k, Clinical chemistry and hematological parameters (measures of sodium (a), potassium (b), chloride (c), anion gap (d), bicarbonate (e), blood urea nitrogen (f), glucose ( $(\mathbf{g})$, calcium (h), hematocrit (i), hemoglobin (j) and

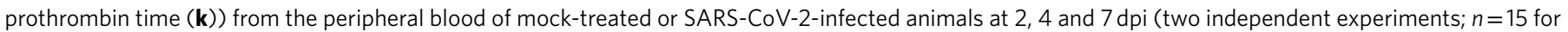
naive mice; $n=12$ for $2 \mathrm{dpi} ; n=15$ for $4 \mathrm{dpi} ; n=15$ for $7 \mathrm{dpi}$; one-way ANOVA with Dunnett's test; $P$ values: ${ }^{\star \star \star} P=0.0009$ (c); $P=0.0237$ and ${ }^{\star \star \star \star} P=0.0001$ (d); ${ }^{\star \star \star \star} P<0.0001(\mathbf{e}, \mathbf{i}-\mathbf{k})$ ). I, Serum D-dimer levels of mock-treated or SARS-CoV-2-infected animals at 2, 4 and $7 \mathrm{dpi}$ (two independent experiments; $n=5$ for naive mice; $n=5$ for $2 \mathrm{dpi} ; n=6$ for $4 \mathrm{dpi} ; n=5$ for $7 \mathrm{dpi}$; one-way ANOVA with Dunnett's test; ${ }^{\star} P=0.0246$ ). $\mathbf{m}$. Results of a treadmill performance test, as measured by the distance traveled in $6 \mathrm{~min}$ (two independent experiments; $n=6$ for naive mice; $n=6$ for $2 \mathrm{dpi}$; $=8$ for $4 \mathrm{dpi} ; n=6$ for $7 \mathrm{dpi}$; two-way ANOVA; $\left.{ }^{\star \star \star \star} P<0.0001\right) . \mathbf{n}-\mathbf{w}$, Respiratory mechanics parameters (respiratory system resistance (n), respiratory system elastance (o), inspiratory capacity $(\mathbf{p})$, quasistatic compliance $(\mathbf{q})$, pressure-volume loops ( $\mathbf{r})$, shape parameter $K(\mathbf{s})$, hysteresis area (t), Newtonian resistance (u), tissue damping ( $\mathbf{v})$ and tissue elastance $(\mathbf{w})$ ) from the lung function assessment in mock-treated or SARS-CoV-2-infected male and female mice at 2 , 4 and 7 dpi. Individual results with group means from two independent experiments are shown ( $n=6$ for naive mice; $n=6$ for $2 \mathrm{dpi} ; n=8$ for $4 \mathrm{dpi} ; n=6$ for $7 \mathrm{dpi}$; one-way ANOVA with Dunnett's test; $P$ values: ${ }^{\star \star \star} P=0.0001(\mathbf{n}) ;{ }^{\star \star \star} P=0.0002(\mathbf{o}) ;{ }^{\star \star} P=0.0024(\mathbf{p}) ;{ }^{\star \star \star} P=0.0008(\mathbf{q}) ;{ }^{\star \star} P=0.0015(\mathbf{s}) ;{ }^{\star \star} P=0.0044(\mathbf{t}) ;{ }^{\star \star \star} P=0.0002(\mathbf{v}) ;$ $\star \star \star P=0.0003(\mathbf{w}))$. Bars represent means and dotted horizontal lines indicate the mean value of the mock-infected group. Asterisks indicate statistical significance as compared with mock infection. In $\mathbf{m}$ and $\mathbf{r}$, symbols represent means \pm s.e.m.

conducting airways, which is consistent with both our histopathological analysis in mice and measurements of pulmonary function in humans with viral pneumonia and respiratory failure including COVID-19 (ref. ${ }^{17}$ ).
The immune response to SARS-CoV-2 infection. An excessive pro-inflammatory host response to SARS-CoV-2 infection is hypothesized to contribute to pulmonary pathology and the development of respiratory distress in some patients with COVID-19 
(ref. ${ }^{18}$ ). To evaluate the composition of the immune cell response in SARS-CoV-2-infected K18-hACE2 mice, we performed flow cytometric analysis on lung homogenates and bronchoalveolar lavage (BAL) fluid at three time points after intranasal virus inoculation (Fig. 4a,b and Extended Data Fig. 3a). Consistent with our histopathological analysis, numbers of $\mathrm{CD} 45^{+}$immune cells trended higher in the BAL beginning at $2 \mathrm{dpi}$ and in the lung at $4 \mathrm{dpi}$, although these differences did not attain statistical significance. The cellular infiltrates at 4 and $7 \mathrm{dpi}$ in the lung were composed principally of myeloid cell subsets including $\mathrm{Ly} 6 \mathrm{G}^{+}$neutrophils, Ly6C $\mathrm{C}^{+}$ monocytes and $\mathrm{CD} 11 \mathrm{~b}^{+} \mathrm{CD} 11 \mathrm{c}^{+}$dendritic cells. In the BAL fluid, monocyte numbers peaked at $4 \mathrm{dpi}$ and levels of neutrophils and dendritic cells continued to rise through $7 \mathrm{dpi}$. Accumulation of monocytes in the BAL fluid coincided with a decrease in the number of tissue-resident alveolar macrophages-an observation consistent with single-cell RNA sequencing (RNA-seq) analysis of BAL fluid of patients with severe COVID-19 disease ${ }^{19,20}$. By 7 dpi, we also observed an increase in several lymphoid cell subsets in the lung, including NK1.1 $1^{+}$natural killer cells, $\gamma \delta \mathrm{CD}^{+}{ }^{+} \mathrm{T}$ cells, $\mathrm{CD}^{+} \mathrm{CD} 4^{+}$ $\mathrm{T}$ cells, $\mathrm{CD}^{+}{ }^{+} \mathrm{CD} 8^{+} \mathrm{T}$ cells and activated $\mathrm{CD} 44^{+} \mathrm{CD} 3^{+} \mathrm{CD} 8^{+} \mathrm{T}$ cells (Fig. 4a).

Extensive changes in cytokine profiles are associated with COVID-19 disease progression ${ }^{21-23}$. Compared with the lungs of uninfected K18-hACE2 control mice, we observed induction of Ifnb, Il28, Ifng, Cxcl10, Cxcl11 and Ccl2 messenger RNA (mRNA) over the first week (Fig. 4c), with the highest expression occurring at $2 \mathrm{dpi}$ for all cytokines except Ifng and $\mathrm{Ccl} 2$. We also measured protein levels in the lungs using a multiplex assay of 44 different cytokines and chemokines (Fig. 4d and Extended Data Fig. 4). Although mRNA expression was highest at $2 \mathrm{dpi}$, almost all upregulated pro-inflammatory cytokines (interferon- $\beta$ (IFN- $\beta$ ), interleukin-6 (IL-6), C-X-C motif chemokine ligand 10 (CXCL10), CXCL9, chemokine ligand 5 (CCL5), CCL12, tissue inhibitor of metalloproteinase 1, tumor necrosis factor- $\alpha$ (TNF- $\alpha$ ) and granulocyte colony-stimulating factor (G-CSF)), T cell-associated cytokines (IL-10, IFN- $\gamma$ and IL-2) and myeloid cell-associated chemokines (CCL2, CCL3, CCL4, CXCL1 and leukemia inhibitory factor) peaked at $7 \mathrm{dpi}$. These data are consistent with cytokine profiling of serum from human patients with COVID-19 and transcriptional analysis of the BAL fluid of human patients, which showed that elevated levels of IL-10, IL-6, IL-2, IL-7, G-CSF, CXCL10, CCL2, CCL3 and TNF- $\alpha$ correlate with disease severity ${ }^{20,24-26}$. Overall, our data suggest that, in the context of the inflammatory response to SARS-CoV-2 in the lungs of K18-hACE2 mice, many cytokines and chemokines are induced, with some having sustained expression and others showing rapid up- and downregulation patterns.

Lymphopenia-particularly T cell lymphopenia-has been associated with severe COVID-19 in humans ${ }^{27-29}$. In K18-hACE2 mice,
SARS-CoV-2 infection resulted in a markedly decreased number of $\mathrm{B}$ cells, $\mathrm{CD}^{+} \mathrm{T}$ cells, CD8 ${ }^{+} \mathrm{T}$ cells and monocytes in peripheral blood at 5 dpi (Fig. 4e and Extended Data Fig. 3b). An increased neutrophil-to-lymphocyte ratio was also observed (Fig. 4f), which is seen in human patients ${ }^{30}$. Of note, decreased lymphocyte numbers were observed with different inoculating doses of SARS-CoV-2 and were present, even in mice receiving only $10^{2}$ p.f.u., before the beginning of weight loss at $6 \mathrm{dpi}$.

Distinct transcriptional signatures are associated with early and late immune responses to SARS-CoV-2 infection. Studies in other small animals and humans have reported cytokine signatures coupled with delayed type I IFN signaling or elevated IFN signatures in the $\operatorname{lung}^{31,32}$. To assess how the kinetics of infection and ensuing inflammation modulate the cytokine and IFN response to SARS-CoV-2, we performed RNA-seq of lung homogenates of K18-transgenic mice at 0 (mock), 2, 4 and $7 \mathrm{dpi}$. Principal component analysis revealed distinct transcriptional signatures that were associated, at $7 \mathrm{dpi}$ (Fig. 5a), with overlapping signatures at 2 and $4 \mathrm{dpi}$. Hundreds of genes were differentially expressed at all time points compared with mock-infected animals (Fig. 5b), many of which were associated with IFN signaling, nuclear factor-kB-dependent cytokine responses or leukocyte activation. In agreement with the principal component analysis, only 449 differentially expressed genes were shared at all time points when compared with the mock. In contrast, 1,975 unique differentially expressed genes were identified between the mock and $7 \mathrm{dpi}$, whereas only 59 and 152 genes were different between the mock and 2 and 4 dpi, respectively (Fig. $5 \mathrm{c}$ ). Gene Ontology analysis of the top upregulated genes at all time points showed enrichment of gene clusters in cytokine-mediated signaling, type I and II IFN signaling, neutrophil activation and pathogen recognition receptor signaling (Fig. 5d). Upregulation of gene sets involved in cytokine-mediated signaling, neutrophil activation, cellular responses to type II IFN and Toll-like receptor signaling were most pronounced at $7 \mathrm{dpi}$ (Fig. $5 \mathrm{e}-\mathrm{g}$, Extended Data Fig. $5 \mathrm{a}-\mathrm{c}$ and Supplementary Table 1). Of note, different genes in the type I IFN signaling pathway were upregulated at 2 and $4 \mathrm{dpi}$ (for example, Irf9, Irf7, Stat1 and certain IFN-stimulated genes (ISGs): Isg15, $M x 1$, Oas3, Ifit1, Ifit2 and Ifit3) versus $7 \mathrm{dpi}$ (for example, Ifnar1/2, Tyk2, Irf1 and certain ISGs: Samhd1, Oas2 and Ifitm1). This suggests a temporally distinct type I IFN response (Fig. 5f), which has been described previously with IFN- $\alpha$ and IFN- $\beta$ subtypes ${ }^{33-35}$. Alternatively, the differences in IFN and ISG signatures at early and late time points could reflect differential signaling contributions of type I, II and III IFNs, as these cytokines are both expressed in the lung after SARS-CoV-2 infection ${ }^{36}$ and induce overlapping yet non-identical sets of ISGs ${ }^{37}$. Collectively, the RNA-seq data from the lungs of K18-hACE2 mice show distinct immune signatures

Fig. 4 | Immune response to SARS-CoV-2 infection in the lungs of K18-hACE2 mice. a,b, Flow cytometric analysis of lung tissues (a) and BAL (b) at 2, 4 and $7 \mathrm{dpi}$ with SARS-CoV-2 (two independent experiments per group; $n=4$ for naive mice; $n=7$ for $2 \mathrm{dpi} ; n=8$ for $4 \mathrm{dpi} ; n=8$ for $7 \mathrm{dpi}$; one-way ANOVA; $P$ values: ${ }^{\star} P=0.0235,{ }^{\star \star} P=0.02$ and ${ }^{\star \star \star} P=0.005(\mathbf{a}) ;{ }^{*} P=0.0465$ and ${ }^{\star \star} P=0.0062(\mathbf{b})$ ). Bars represent mean values. Asterisks indicate statistical significance as compared with mock infection. DCs, dendritic cells; NK, natural killer. c, Fold change in the gene expression of the indicated cytokines and chemokines, as determined by RT-qPCR, normalized to Gapdh and compared with naive controls in lung homogenates at 2, 4 and $7 \mathrm{dpi}$ (two independent experiments; $n=11$ for $2 \mathrm{dpi} ; n=15$ for $4 \mathrm{dpi} ; n=16$ for $7 \mathrm{dpi}$ ). Dotted horizontal lines indicate the average level of cytokine or chemokine transcript in naive mice. d, Heat maps of cytokine levels, as measured by multiplex platform, in the lung tissues of SARS-CoV-2-infected mice at 2, 4 and 7 dpi. For each cytokine, the fold change was calculated as compared with mock-infected animals and the $\log _{2}$ [fold change] was plotted in the corresponding heat map (two independent experiments; $n=9$ for naive mice; $n=12$ for $2 \mathrm{dpi} n=13$ for $4 \mathrm{dpi}, n=12$ for $7 \mathrm{dpi}$; associated statistics are reported in Extended Data Fig. 5 ). EPO, erythropoetin; GM-CSF, granulocyte-macrophage colony-stimulating factor; LIF, leukemia inhibitory factor; M-CSF, macrophage colony-stimulating factor; TIMP-1, tissue inhibitor of metalloproteinase 1; VEGF, vascular endothelial growth factor. e,f, Flow cytometric analysis of peripheral blood at 5 dpi in 6-week-old male and female mice following intranasal inoculation with the indicated doses of SARS-CoV-2 or mock infection. e, Cell numbers per ml blood (one independent experiment; $n=3$ for naive; $n=3$ for $10^{2}$ p.f.u.; $n=4$ for $10^{3}$ p.f.u.; $n=4$ for $10^{4}$ p.f.u.; $n=4$ for $10^{5}$ p.f.u.; one-way ANOVA; $P$ values: ${ }^{\star} P=0.0385,{ }^{\star \star} P=0.0026$ and ${ }^{\star \star \star}{ }^{\star} P=0.0006$ (B cells); ${ }^{\star} P=0.0259$ and ${ }^{\star \star} P=0.002$ (CD4 ${ }^{+}$T cells); ${ }^{\star} P=0.0284,{ }^{\star \star} P=0.0020$ and ${ }^{\star \star \star} P=0.0002\left(\mathrm{CD} 8{ }^{+}\right.$ T cells); ${ }^{\star} P=0.0129$ and ${ }^{\star \star} P=0.0068$ (monocytes)). Bars indicate mean values. f, Frequency of lymphocytes, monocytes and neutrophils (one independent experiment; $n=3$ for naive mice; $n=3$ for $10^{2}$ p.f.u.; $n=4$ for $10^{3}$ p.f.u.; $n=4$ for $10^{4}$ p.f.u.; $n=4$ for $10^{5}$ p.f.u.). Bars indicate means \pm s.e.m. 
associated with early (days 2 and 4) and late (day 7) SARS-CoV-2 infection.

\section{Discussion}

In this study, we found that SARS-CoV-2 infection of K18-hACE2-transgenic mice causes severe pulmonary disease. After intranasal SARS-CoV-2 inoculation, K18-hACE2 mice rapidly lost weight after $4 \mathrm{dpi}$ and began to succumb to disease at 7 dpi. High levels of viral RNA and infectious virus were detected in the lungs of infected animals at 2, 4 and $7 \mathrm{dpi}$ by RT-qPCR, in situ hybridization and plaque assay. Infection was accompanied by high levels of pro-inflammatory cytokines and chemokines in the lung and a cellular infiltrate primarily comprising monocytes, neutrophils and $\mathrm{T}$ cells. The combined infection and inflammation
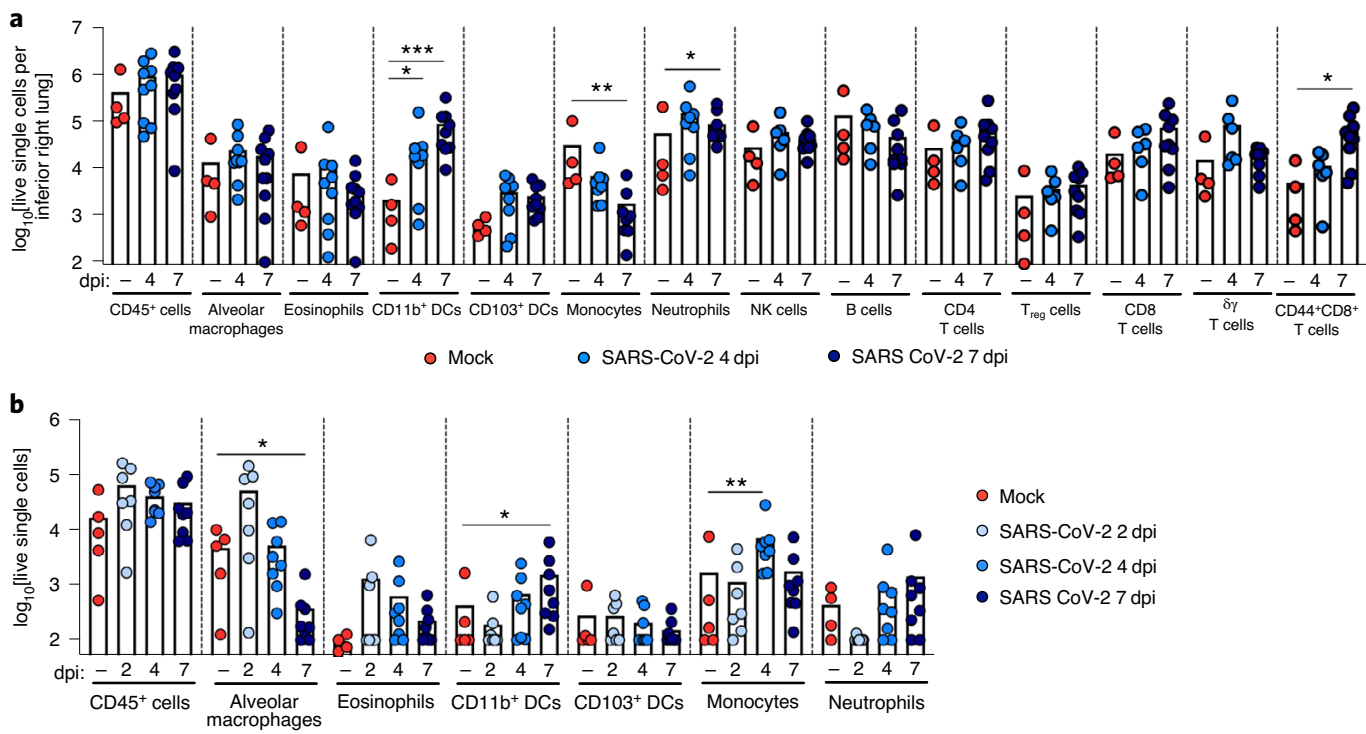
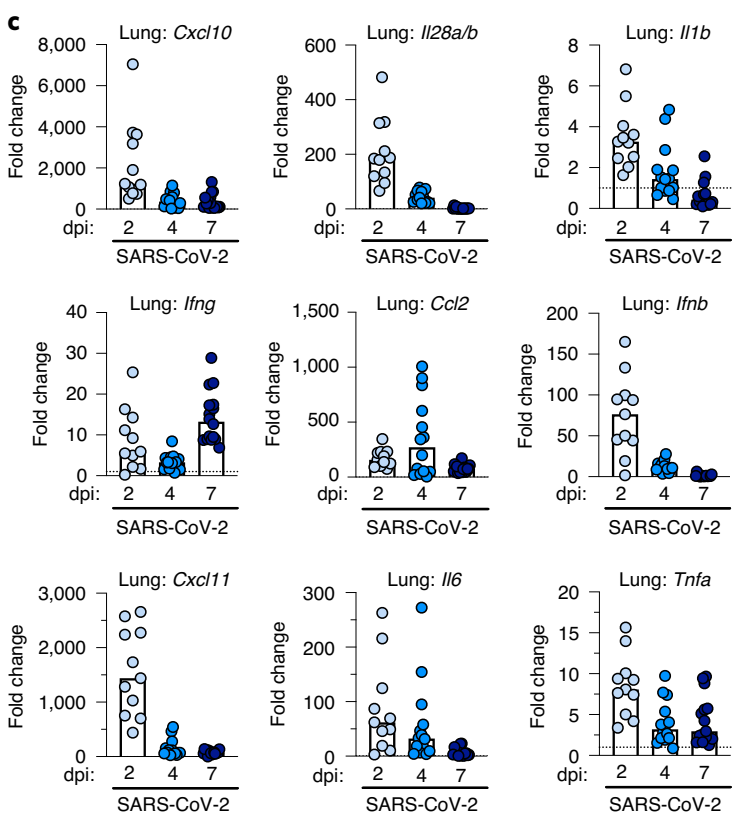
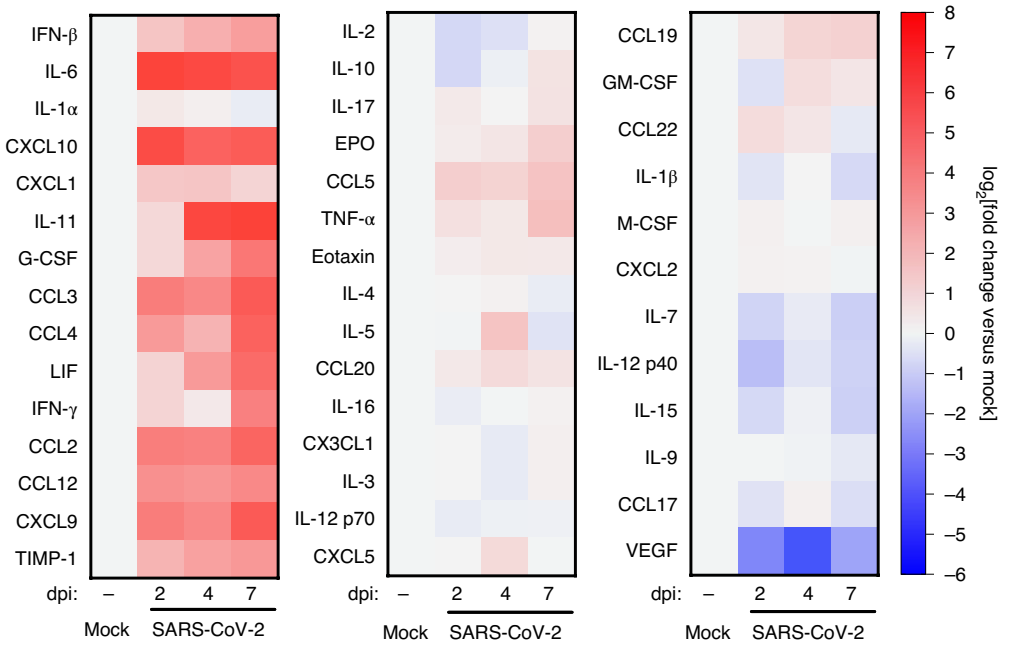
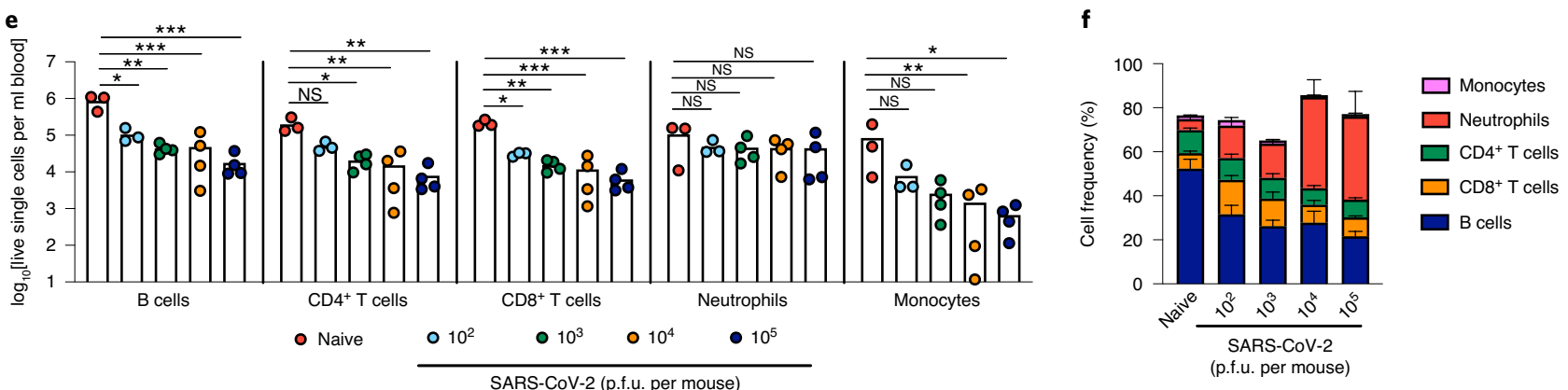

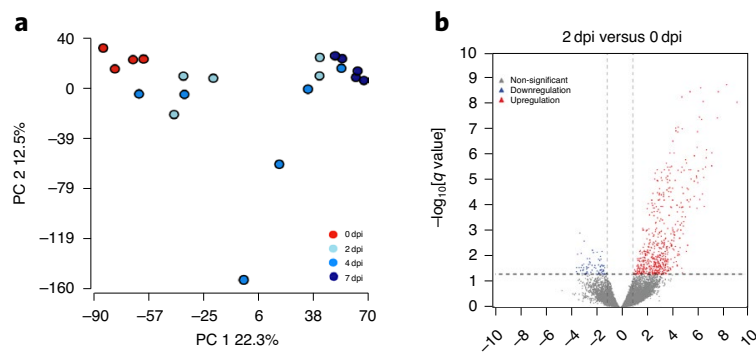

$\log _{2}$ [fold change] d

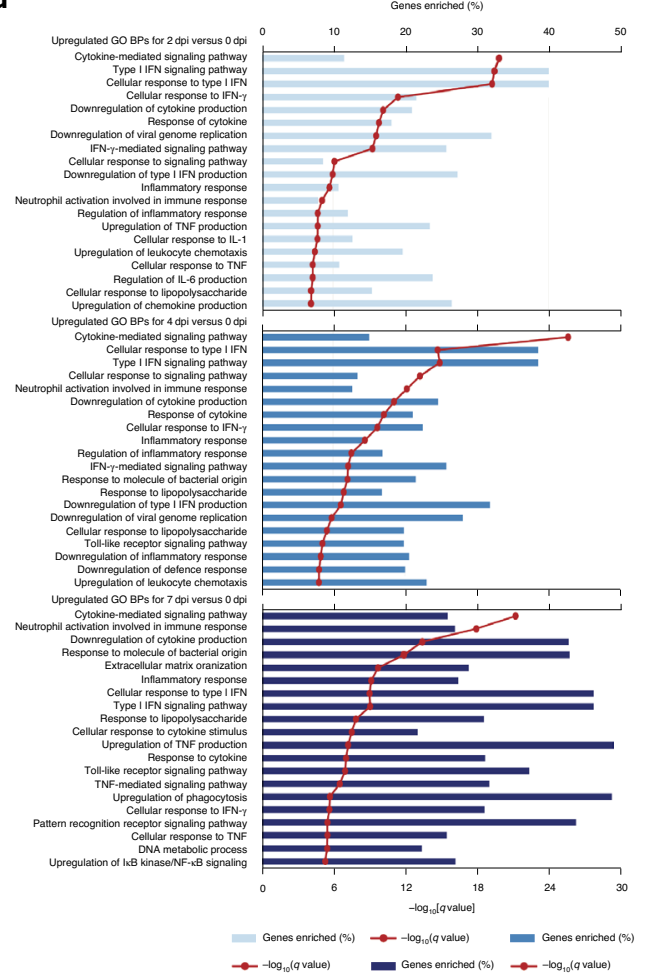

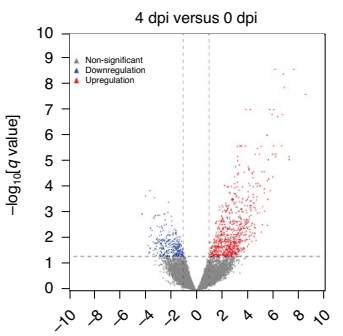

$\log _{2}$ [fold change]

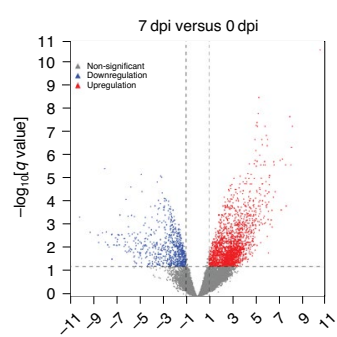

$\log _{2}$ [fold change]
C

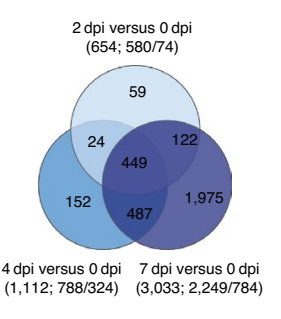

.

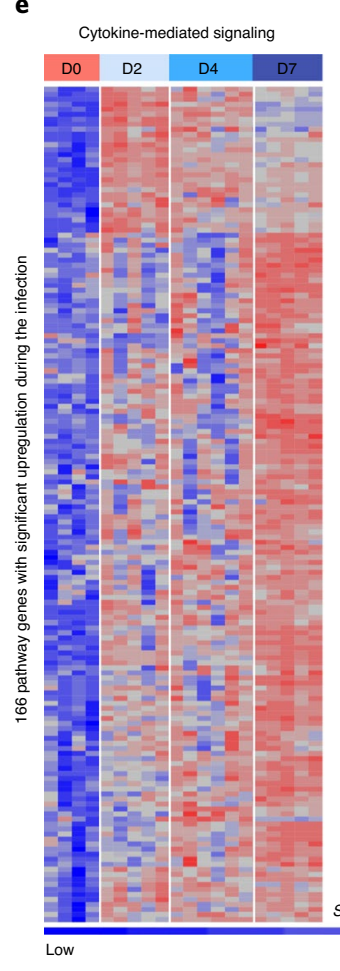

f

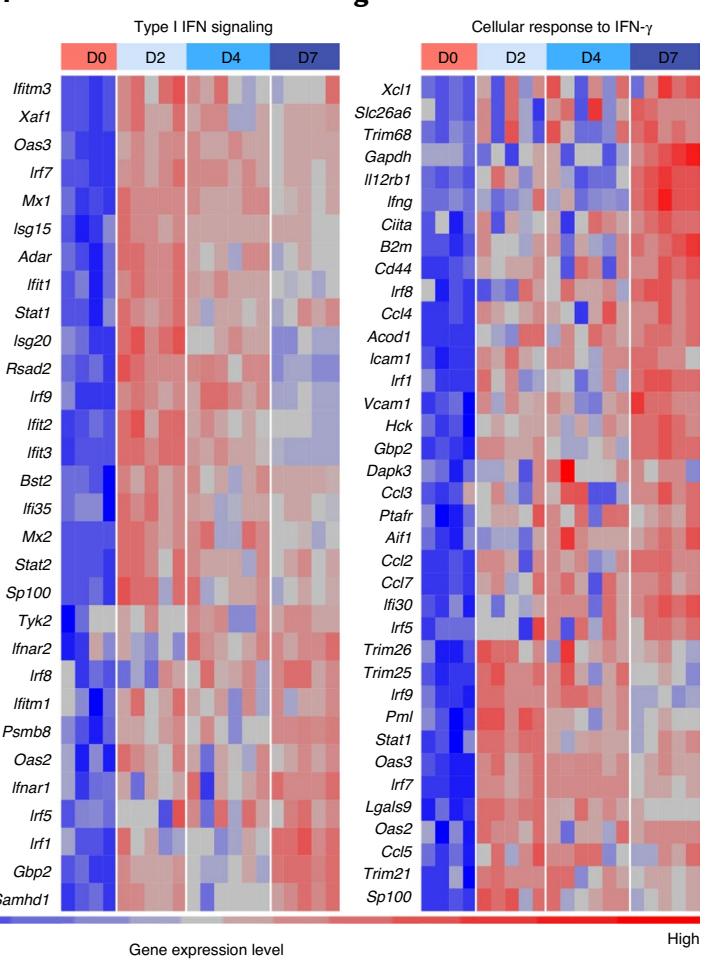

g

Fig. 5 | Distinct transcriptional signatures are associated with early and late immune responses to SARS-CoV-2 infection. a-g, RNA-seq analysis of the lung homogenates of naive K18-hACE2 mice and at 2, 4 and $7 \mathrm{dpi}$ (two independent experiments; $n=4$ for naive mice; $n=5$ for $2 \mathrm{dpi} ; n=6$ for $4 \mathrm{dpi} ; n=5$ for $7 \mathrm{dpi}$ ). a, Principal component analysis was performed for 20 samples with the $\log _{2}$-transformed gene-level counts per million (log 2 [CPM]) data. PC, principal component. b. Volcano plots comparing differentially expressed genes from samples taken at day 2 versus day 0 , day 4 versus day 0 , and day 7 versus day 0 . Red and blue indicate upregulated and downregulated genes, respectively, with a fold change $>2$ and a false discovery rate $(q$ value) $<0.05$. Multiple comparisons were accounted for by calculation of a Benjamini-Hochberg false discovery rate-adjusted $P$ value (that is, $q$ value). Dashed horizontal lines mark a $q$ value of 0.05 and dashed vertical lines indicate a $\log _{2}$ [fold change] of 1 and -1 . Each dot in the volcano plots represents a single gene. $\mathbf{c}$, Venn diagram of overlapping genes identified in differential expression analysis when comparing the mock treatment with 2, 4 and $7 \mathrm{dpi}$. Numbers in parentheses under each comparison indicate the total number of significantly differential genes followed by the proportion of the total that are up- and downregulated. d, Gene Ontology (GO) enrichment analysis of biological process (BP) terms enriched in upregulated genes from comparisons of mice at 2, 4 and 7 dpi versus mock-infected mice. Terms were ranked by the false discovery rate ( $q$ value) and the top 20 are listed after eliminating redundant terms. Bars show

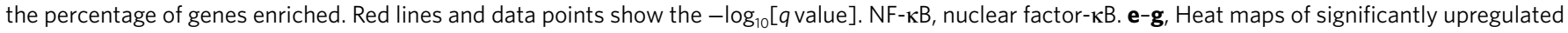
genes during SARS-CoV-2 infection enriched in the cytokine-mediated signaling pathway (e), type I IFN signaling pathway (f) and cellular response to IFN- $\gamma(\mathbf{g})$, identified through Gene Ontology analysis. Genes shown in each pathway are the union of the differentially expressed genes from the three comparisons (2, 4 and 7 dpi versus mock infected). Columns represent samples and rows represent genes. Gene expression levels in the heat maps are z score-normalized values determined from $\log _{2}[C P M]$ values. $D$, day.

resulted in severe interstitial pneumonia characterized by collapsed alveolar spaces. This caused detrimental changes in lung physiology, including decreased exercise tolerance, reduced inspiratory capacity and stiffening of the lung parenchyma.

SARS-CoV-2 infection is subclinical or mild in most human cases. A small yet clinically important fraction develop life-threatening disease requiring hospitalization and intensive care. Mild disease is a feature of SARS-CoV-2 infection in naturally susceptible animals including hamsters, ferrets, cats and non-human primates ${ }^{5}$.
This is perhaps unsurprising given that the strongest risk factors for developing severe COVID-19 in humans (for example, old age, cardiovascular disease and diabetes) are absent in many laboratory animals. Mild to moderate disease is seen in many rodent models of SARS-CoV-2 infection, including those expressing hACE2 via viral vectors or transgenes ${ }^{8,12,13,38}$. Thus, the severity of disease we observed following SARS-CoV-2 infection of K18-hACE2 mice is unique. As the onset of severe clinical disease in K18-hACE2 mice occurs days after peak viral infection and is associated with high 
levels of infiltrating immune cells and inflammatory mediators in the lung, immune responses probably contribute to pathogenesis.

The histopathological changes we observed in the infected lungs of K18-hACE2 mice correlate with the impaired pulmonary function. Pneumocytes became infected early, which led to recruitment of leukocytes into the pulmonary interstitium, production of pro-inflammatory cytokines, injury to parenchymal cells, collapse of the alveolar space and compromise of gas exchange, all of which could cause the hypercapnia we observed at $7 \mathrm{dpi}$. This course is remarkably consistent with human disease in which rapid early viral replication is followed by inflammatory responses, which are believed to contribute to pathology, morbidity and mortality ${ }^{39}$.

A fundamental understanding of the immunological processes that influence COVID-19 disease is needed to select immunomodulatory interventions that target key cell types or pathways. We saw substantial immune cell accumulation in the lungs of K18-hACE2 mice-an observation consistent with postmortem analysis of human patients ${ }^{40}$. Lung infiltrates were composed primarily of myeloid cells, including monocytes and neutrophils as well as activated $\mathrm{CD}^{+} \mathrm{T}$ cells, and corresponded with high levels of chemokines that drive their migration. The lymphopenia associated with severe COVID-19 in humans is attributed in part to the immune cell migration into inflamed tissues ${ }^{22,41}$. We also observed lymphopenia in circulating blood cells following SARS-CoV-2 infection, and this often preceded weight loss. This result is consistent with human studies that correlate lymphopenia with the development of severe COVID-19 (ref. ${ }^{42}$ ). In transcriptional analyses of BAL fluid from infected humans with severe COVID-19, an accumulation of $\mathrm{CD}^{+} \mathrm{T}$ cells, neutrophils and monocytes coincided with the loss of alveolar macrophages ${ }^{19,20}$. In our study, using cytokine analysis and RNA-seq of lung homogenates, we detected enhanced expression of several myeloid cell chemoattractants (for example, CCL2, CCL3, CCL4, CXCL1 and CXCL10) and other key inflammatory cytokines (TNF- $\alpha$, IL-6 and G-CSF) that correlate with COVID-19 disease severity in humans ${ }^{31,32}$. Given these parallel findings, studies in K18-hACE2 mice evaluating the role of specific immune pathways and cell subsets in disease pathogenesis could inform the selection of immunomodulatory agents for severe COVID-19.

The role of type I IFN in SARS-CoV-2 pathogenesis in this model warrants further investigation as it has been suggested that a dysregulated type I IFN response contributes to excessive immunopathology. Indeed, in SARS-CoV infection, type I IFN signaling appears to be pro-inflammatory and not antiviral ${ }^{43}$. Our RNA-seq analysis revealed differences in the type I IFN gene signatures associated with early and late SARS-CoV-2 infection. Given that ISGs can exert diverse functions apart from their antiviral activities, including inflammatory, metabolic and transcriptional effects ${ }^{44}$, these two early and late ISG modules may have different functional consequences following SARS-CoV-2 infection. Furthermore, how these temporally distinct programs are induced and regulated remains uncertain and may be the result of cell type specificity, kinetics and sensitivity to different type I or III IFN subtypes.

The development of severe disease following SARS-CoV-2 infection is an important feature of the K18-hACE2 model, although the precise reason for this susceptibility compared with other hACE2-transgenic models remains unknown. Potential explanations include a high number of hACE2 transgene insertions (https://www.jax.org/strain/034860) and/or the strength and cellular specificity of the K18 promoter. Human ACE2 is expressed in multiple tissues in the K18-hACE2 mouse ${ }^{10}$, which could allow for SARS-CoV-2 infection of multiple organs. While we detected viral RNA in several tissues, the lung was the most heavily infected and showed the most consistent and severe histopathological changes; these findings were anticipated given the known tropism of SARS-CoV-2 for the respiratory tract and the intranasal route of infection. Moderate levels of viral RNA were also found in the heart, kidney and spleen, with peak titers at 2 and $4 \mathrm{dpi}$, whereas levels in gastrointestinal tract tissues (duodenum, ileum and colon) were lower. In the gastrointestinal tract of K18-hACE2 mice, hACE2 was expressed most abundantly in the colon, which correlated with infection seen at later time points. Although hACE2 is expressed in the gastrointestinal tract in other hACE2-expressing mice, productive infection was observed only upon intragastric inoculation or at early time points following intranasal infection ${ }^{12,13}$.

We observed dichotomous SARS-CoV-2 infection in the brain, with high virus levels in approximately $40 \%$ of mice at $7 \mathrm{dpi}$ and low levels in the remaining $60 \%$ of animals. Infection of the brain also was observed in K18-hACE2 mice infected with SARS-CoV ${ }^{10,15}$ but occurred earlier (at 3 to $4 \mathrm{dpi}$ ) and more uniformly. Similar to the experiments with SARS-CoV, we did not detect SARS-CoV-2 in the olfactory bulb, which suggests that both SARS-CoV and SARS-CoV-2 cross the blood-brain barrier instead of traversing the cribriform plate and infecting neuronal processes near the site of intranasal inoculation ${ }^{45}$. Notwithstanding these data, and unlike SARS-CoV, alterations in smell and taste are features of SARS-CoV-2 infection in humans ${ }^{46}$, suggesting that cell types within the olfactory system may be susceptible to infection or injury. More study is needed to clarify the routes SARS-CoV-2 dissemination throughout the host and particularly how it accesses the brain in some animals and humans.

While SARS-CoV-2 lung infection in K18-hACE2 mice provides a model for studying severe infection that recapitulates features of COVID-19 in humans, we acknowledge several limitations. The expression of the hACE2 transgene is non-physiological in several respects. It is driven by a non-native (that is, K18) promotor, resulting in tissue expression levels that are distinct from endogenously expressed ACE2. The expression of ACE2 in K18-hACE2 mice is independent of the complex regulatory systems that govern ACE2 levels ${ }^{47}$. Whereas in humans the ACE2 gene is located on the $\mathrm{X}$ chromosome, the hACE2 transgene is inserted as multiple copies in mouse chromosome 2 (https://www.jax.org/strain/034860). As such, comorbid conditions (for example, obesity, hypertension, diabetes and sex) that alter ACE2 expression in humans ${ }^{47}$ probably cannot be modeled faithfully in this transgenic mouse.

In summary, we found that SARS-CoV-2 infection of K18-hACE2-transgenic mice supports robust viral replication in the lung, which leads to severe immune cell infiltration, inflammation and pulmonary disease. Thus, the K18-hACE2 mouse is an attractive small animal model for defining the mechanisms of the pathogenesis of severe COVID-19 and may be useful for evaluating countermeasures that reduce virus infection or associated pathological inflammatory responses.

\section{Online content}

Any methods, additional references, Nature Research reporting summaries, source data, extended data, supplementary information, acknowledgements, peer review information; details of author contributions and competing interests; and statements of data and code availability are available at https://doi.org/10.1038/ s41590-020-0778-2.

Received: 9 July 2020; Accepted: 7 August 2020; Published online: 24 August 2020

\section{References}

1. Ziegler, C. G. K. et al. SARS-CoV-2 receptor ACE2 is an interferon-stimulated gene in human airway epithelial cells and is detected in specific cell subsets across tissues. Cell 181, 1016-1035.e19 (2020).

2. Hamming, I. et al. Tissue distribution of ACE2 protein, the functional receptor for SARS coronavirus. A first step in understanding SARS pathogenesis. J. Pathol. 203, 631-637 (2004).

3. Lai, C. C., Ko, W. C., Lee, P. I., Jean, S. S. \& Hsueh, P. R. Extra-respiratory manifestations of COVID-19. Int. J. Antimicrob. Agents https://doi. org/10.1016/j.ijantimicag.2020.106024 (2020). 
4. Rockx, B. et al. Comparative pathogenesis of COVID-19, MERS, and SARS in a nonhuman primate model. Science 368, 1012-1015 (2020).

5. Cleary, S. J. et al. Animal models of mechanisms of SARS-CoV-2 infection and COVID-19 pathology. Br. J. Pharmacol. https://doi.org/10.1111/bph.15143 (2020)

6. Letko, M., Marzi, A. \& Munster, V. Functional assessment of cell entry and receptor usage for SARS-CoV-2 and other lineage B betacoronaviruses. Nat. Microbiol. 5, 562-569 (2020).

7. Wan, Y., Shang, J., Graham, R., Baric, R. S. \& Li, F. Receptor recognition by novel coronavirus from Wuhan: an analysis based on decade-long structural studies of SARS. J. Virol. https://doi.org/10.1128/jvi.00127-20 (2020).

8. Hassan, A. O. et al. A SARS-CoV-2 infection model in mice demonstrates protection by neutralizing antibodies. Cell https://doi.org/10.1016/j. cell.2020.06.011 (2020)

9. Israelow, B. et al. Mouse model of SARS-CoV-2 reveals inflammatory role of type I interferon signaling. J. Exp. Med. 217, e20201241 (2020).

10. McCray, P. B. Jr. et al. Lethal infection of K18-hACE2 mice infected with severe acute respiratory syndrome coronavirus. J. Virol. 81, 813-821 (2007).

11. Jiang, R. D. et al. Pathogenesis of SARS-CoV-2 in transgenic mice expressing human angiotensin-converting enzyme 2. Cell 182, 50-58.e8 (2020).

12. Bao, L. et al. The pathogenicity of SARS-CoV-2 in hACE2 transgenic mice. Nature https://doi.org/10.1038/s41586-020-2312-y (2020).

13. Sun, S. H. et al. A mouse model of SARS-CoV-2 infection and pathogenesis. Cell Host Microbe https://doi.org/10.1016/j.chom.2020.05.020 (2020).

14. Yang, X., Pabon, L. \& Murry, C. E. Engineering adolescence: maturation of human pluripotent stem cell-derived cardiomyocytes. Circ. Res. 114, 511-523 (2014).

15. Netland, J., Meyerholz, D. K., Moore, S., Cassell, M. \& Perlman, S. Severe acute respiratory syndrome coronavirus infection causes neuronal death in the absence of encephalitis in mice transgenic for human ACE2. J. Virol. 82, 7264-7275 (2008).

16. Robichaud, A., Fereydoonzad, L., Urovitch, I. B. \& Brunet, J. D. Comparative study of three flexiVent system configurations using mechanical test loads. Exp. Lung Res. 41, 84-92 (2015).

17. Barbeta, E. et al. SARS-CoV-2-induced acute respiratory distress syndrome: pulmonary mechanics and gas exchange abnormalities. Ann. Am. Thoracic Soc. https://doi.org/10.1513/AnnalsATS.202005-462RL (2020).

18. Giamarellos-Bourboulis, E. J. et al. Complex immune dysregulation in COVID-19 patients with severe respiratory failure. Cell Host Microbe https:// doi.org/10.1016/j.chom.2020.04.009 (2020).

19. Liao, M. et al. Single-cell landscape of bronchoalveolar immune cells in patients with COVID-19. Nat. Med. 26, 842-844 (2020).

20. Bost, P. et al. Host-viral infection maps reveal signatures of severe COVID-19 patients. Cell 181, 1475-1488.e12 (2020).

21. Mehta, P. et al. COVID-19: consider cytokine storm syndromes and immunosuppression. Lancet 395, 1033-1034 (2020).

22. Huang, C. et al. Clinical features of patients infected with 2019 novel coronavirus in Wuhan, China. Lancet 395, 497-506 (2020).

23. Chua, R. L. et al. COVID-19 severity correlates with airway epitheliumimmune cell interactions identified by single-cell analysis. Nat. Biotechnol. https://doi.org/10.1038/s41587-020-0602-4 (2020).

24. Chen, X. et al. Detectable serum SARS-CoV-2 viral load (RNAaemia) is closely correlated with drastically elevated interleukin 6 (IL-6) level in critically ill COVID-19 patients. Clin. Infect. Dis. https://doi.org/10.1093/cid/ ciaa449 (2020).

25. Liu, J. et al. Longitudinal characteristics of lymphocyte responses and cytokine profiles in the peripheral blood of SARS-CoV-2 infected patients. EBioMedicine 55, 102763 (2020).
26. Yang, Y. et al. Plasma IP-10 and MCP-3 levels are highly associated with disease severity and predict the progression of COVID-19. J. Allergy Clin. Immunol. 146, 119-127.e4 (2020).

27. Fu, L. et al. Clinical characteristics of coronavirus disease 2019 (COVID-19) in China: a systematic review and meta-analysis. J. Infect. 80, 656-665 (2020).

28. Liu, R. et al. Decreased T cell populations contribute to the increased severity of COVID-19. Clin. Chim. Acta 508, 110-114 (2020).

29. Wilk, A. J. et al. A single-cell atlas of the peripheral immune response in patients with severe COVID-19. Nat. Med. 26, 1070-1076 (2020).

30. Wang, Y. et al. Characterization of an asymptomatic cohort of SARS-COV-2 infected individuals outside of Wuhan, China. Clin. Infect Dis. https://doi. org/10.1093/cid/ciaa629 (2020).

31. Zhou, Z. et al. Heightened innate immune responses in the respiratory tract of COVID-19 patients. Cell Host Microbe 27, 883-890.e2 (2020).

32. Blanco-Melo, D. et al. Imbalanced host response to SARS-CoV-2 drives development of COVID-19. Cell 181, 1036-1045.e9 (2020).

33. Cook, L. E. et al. Distinct roles of interferon alpha and beta in controlling Chikungunya virus replication and modulating neutrophil-mediated inflammation. J. Virol. 94, e00841-19 (2019).

34. Ng, C. T., Mendoza, J. L., Garcia, K. C. \& Oldstone, M. B. Alpha and beta type 1 interferon signaling: passage for diverse biologic outcomes. Cell 164, 349-352 (2016)

35. Ng, C. T. et al. Blockade of interferon beta, but not interferon alpha, signaling controls persistent viral infection. Cell Host Microbe 17, 653-661 (2015).

36. Park, A. \& Iwasaki, A. Type I and type III interferons-induction, signaling, evasion, and application to combat COVID-19. Cell Host Microbe 27, 870-878 (2020).

37. Lazear, H. M., Schoggins, J. W. \& Diamond, M. S. Shared and distinct functions of type I and type III interferons. Immunity 50, 907-923 (2019).

38. Jiang, D. et al. Identification of three interferon-inducible cellular enzymes that inhibit the replication of hepatitis C virus. J. Virol. 82, 1665-1678 (2008).

39. Pan, F. et al. Time course of lung changes at chest CT during recovery from coronavirus disease 2019 (COVID-19). Radiology 295, 715-721 (2020).

40. Xu, Z. et al. Pathological findings of COVID-19 associated with acute respiratory distress syndrome. Lancet Resp. Med. 8, 420-422 (2020).

41. Chen, G. et al. Clinical and immunological features of severe and moderate coronavirus disease 2019. J. Clin. Invest. 130, 2620-2629 (2020).

42. Tan, L. et al. Lymphopenia predicts disease severity of COVID-19: a descriptive and predictive study. Signal Transduct. Target. Ther. 5, 33 (2020); erratum 5, 61 (2020).

43. Channappanavar, R. et al. Dysregulated type I interferon and inflammatory monocyte-macrophage responses cause lethal pneumonia in SARS-CoV-infected mice. Cell Host Microbe 19, 181-193 (2016).

44. Mostafavi, S. et al. Parsing the interferon transcriptional network and its disease associations. Cell 164, 564-578 (2016).

45. Barnett, E. M., Cassell, M. D. \& Perlman, S. Two neurotropic viruses, herpes simplex virus type 1 and mouse hepatitis virus, spread along different neural pathways from the main olfactory bulb. Neuroscience 57, 1007-1025 (1993).

46. Spinato, G. et al. Alterations in smell or taste in mildly symptomatic outpatients with SARS-CoV-2 infection. J. Am. Med. Assoc. 323, 2089-2090 (2020)

47. Li, Y., Zhou, W., Yang, L. \& You, R. Physiological and pathological regulation of ACE2, the SARS-CoV-2 receptor. Pharmacol. Res. 157, 104833 (2020).

Publisher's note Springer Nature remains neutral with regard to jurisdictional claims in published maps and institutional affiliations.

(c) The Author(s), under exclusive licence to Springer Nature America, Inc. 2020 


\section{Methods}

Cells and viruses. Vero E6 (CRL-1586; American Type Culture Collection), Vero CCL81 (American Type Culture Collection) and Vero-furin cells ${ }^{48}$ were cultured at $37^{\circ} \mathrm{C}$ in Dulbecco's modified Eagle's medium (DMEM) supplemented with $10 \%$ fetal bovine serum (FBS), $10 \mathrm{mM}$ HEPES ( $\mathrm{pH} 7.3$ ), $1 \mathrm{mM}$ sodium pyruvate, $1 \times$ non-essential amino acids and $100 \mathrm{U} \mathrm{ml}^{-1}$ penicillin-streptomycin. The 2019n-CoV/USA_WA1/2019 isolate of SARS-CoV-2 was obtained from the US Centers for Disease Control. Infectious stocks were grown by inoculating Vero CCL81 cells and collecting supernatant upon observation of cytopathic effect; debris were removed by centrifugation and passage through a $0.22-\mu \mathrm{m}$ filter. Supernatant was then aliquoted and stored at $-80^{\circ} \mathrm{C}$.

Biosafety. All aspects of this study were approved by the office of Environmental Health and Safety at Washington University School of Medicine before initiation of this study. Work with SARS-CoV-2 was performed in a biosafety level 3 laboratory by personnel equipped with powered air-purifying respirators.

Mice. Animal studies were carried out in accordance with the recommendations in the Guide for the Care and Use of Laboratory Animals of the National Institutes of Health. The protocols were approved by the Institutional Animal Care and Use Committee at the Washington University School of Medicine (assurance number A3381-01). Virus inoculations were performed under anesthesia that was induced and maintained with ketamine hydrochloride and xylazine, and all efforts were made to minimize animal suffering.

Heterozygous K18-hACE c57BL/6J mice (strain: 2B6.Cg-Tg(K18-ACE2) $2 \mathrm{Prlmn} / \mathrm{J})$ were obtained from The Jackson Laboratory. Animals were housed in groups and fed standard chow diets. Mice of different ages and both sexes were administered $2.5 \times 10^{4}$ p.f.u. SARS-CoV-2 via intranasal administration.

Plaque-forming assay. Vero-furin cells $\mathrm{s}^{48}$ were seeded at a density of $2.5 \times 10^{5}$ cells per well in flat-bottom 12-well tissue culture plates. The following day, media was removed and replaced with $200 \mu \mathrm{l}$ of tenfold serial dilutions of the material to be titered, diluted in DMEM + 2\% FBS. One hour later, $1 \mathrm{ml}$ methylcellulose overlay was added. Plates were incubated for $72 \mathrm{~h}$, then fixed with $4 \%$ paraformaldehyde (final concentration) in phosphate-buffered saline (PBS) for $20 \mathrm{~min}$. Plates were stained with $0.05 \%$ (wt/vol) crystal violet in $20 \%$ methanol and washed twice with distilled, deionized water.

\section{Measurement of viral burden and $\boldsymbol{h A C E} 2$ expression. Tissues were weighed} and homogenized with zirconia beads in a MagNA Lyser instrument (Roche Life Science) in $1 \mathrm{ml}$ of DMEM media supplemented with $2 \%$ heat-inactivated FBS. Tissue homogenates were clarified by centrifugation at 10,000 r.p.m. for $5 \mathrm{~min}$ and stored at $-80^{\circ} \mathrm{C}$. RNA was extracted using the MagMAX mirVana Total RNA Isolation Kit (Thermo Fisher Scientific) on the KingFisher Flex extraction robot (Thermo Fisher Scientific). RNA was reverse transcribed and amplified using the TaqMan RNA-to-CT 1-Step Kit (Thermo Fisher Scientific). Reverse transcription was carried out at $48^{\circ} \mathrm{C}$ for $15 \mathrm{~min}$ followed by $2 \mathrm{~min}$ at $95^{\circ} \mathrm{C}$. Amplification was accomplished over 50 cycles as follows: $95^{\circ} \mathrm{C}$ for $15 \mathrm{~s}$ and $60^{\circ} \mathrm{C}$ for $1 \mathrm{~min}$. Copies of SARS-CoV-2 nucleocapsid (N) gene RNA in samples were determined using a previously published assay ${ }^{8}$. Briefly, a TaqMan assay was designed to target a highly conserved region of the $\mathrm{N}$ gene (forward primer: ATGCTGCAATCGTGCTACAA; reverse primer: GACTGCCGCCTCTGCTC; probe: /56-FAM/TCAAGGAAC/ZEN/ AACATTGCCAA/3IABkFQ/). This region was included in an RNA standard to allow for copy number determination down to ten copies per reaction. The reaction mixture contained final concentrations of primers and probe of 500 and $100 \mathrm{nM}$, respectively.

For hACE2 expression, RNA was DNase treated (Thermo Fisher Scientific) following the manufacturer's protocol. RNA levels were quantified as described above with the primer/probe set for hACE2 (IDT assay: Hs.PT.58.27645939), compared with an RNA standard curve and normalized to mg of tissue.

Cytokine and chemokine mRNA measurements. RNA was isolated from lung homogenates as described above. Complementary DNA (cDNA) was synthesized from DNAse-treated RNA using the High-Capacity cDNA Reverse Transcription Kit (Thermo Fisher Scientific) with the addition of RNase inhibitor following the manufacturer's protocol. Cytokine and chemokine expression was determined using TaqMan Fast Universal PCR Master Mix (Thermo Fisher Scientific) with commercial primers/probe sets specific for IFNg (IDT: Mm.PT.58.41769240), IL6 (Mm.PT.58.10005566), IL1b (Mm.PT.58.41616450), TNFa (Mm.PT.58.12575861) CXCL10 (Mm.PT.58.43575827), CCL2 (Mm.PT.58.42151692), CCL5 (Mm.

PT.58.43548565), CXCL11 (Mm.PT.58.10773148.g), IFNb (Mm.PT.58.30132453.g) and $I L 28 a / b$ (Thermo Fisher Scientific; Mm04204156 gH) and the results were normalized to GAPDH (Mm.PT.39a.1) levels. Fold changes were determined using the $2^{-\Delta \Delta \mathrm{Ct}}$ method comparing treated mice with naive controls.

Cytokine and chemokine protein measurements. Lung homogenates were incubated with Triton X-100 ( $1 \%$ final concentration) for $1 \mathrm{~h}$ at room temperature to inactivate SARS-CoV-2. Homogenates then were analyzed for cytokines and chemokines by Eve Technologies using their Mouse Cytokine Array/Chemokine Array 44-Plex (MD44) platform.
Histology and RNA in situ hybridization. Animals were euthanized before harvest and fixation of tissues. The left lung was first tied off at the left main bronchus and collected for viral RNA analysis. The right lung was then inflated with $\sim 1.2 \mathrm{ml} 10 \%$ neutral-buffered formalin (NBF) using a 3 -ml syringe and catheter inserted into the trachea. For harvesting of brains for fixation, the mouse was decapitated and the skull cap removed. The whole brain was removed intact, cut mid-sagittally to increase the surface area of fixation and drop-fixed in $10 \%$ NBF. For the kidney, spleen, liver and heart, organs were removed and drop-fixed in $10 \%$ NBF. For fixation after infection, organs were kept in a $40-\mathrm{ml}$ suspension of NBF for $7 \mathrm{~d}$ before further processing. Tissues were embedded in paraffin and sections were stained with hematoxylin and eosin. RNA in situ hybridization was performed using the RNAscope 2.5 HD Assay (Brown Kit) according to the manufacturer's instructions (Advanced Cell Diagnostics). Briefly, sections were deparaffinized and treated with $\mathrm{H}_{2} \mathrm{O}_{2}$ and Protease Plus before probe hybridization. Probes specifically targeting hACE2 (catalog number 848151) or SARS-CoV-2 spike sequence (catalog number 848561 ) were hybridized followed by proprietary signal amplification and detection with 3,3'-diaminobenzidine. Tissues were counterstained with Gill's hematoxylin. An uninfected mouse was used as a negative control and stained in parallel. Tissue sections were visualized using a Nikon Eclipse microscope equipped with an Olympus DP71 camera, a Leica DM6B microscope equipped with a Leica DFC7000T camera, or an Olympus BX51 microscope with an attached camera.

Flow cytometry analysis of immune cell infiltrates. For analysis of BAL fluid, mice were sacrificed by ketamine overdose, followed by cannulation of the trachea with a 19-G canula. BAL was performed with three washes of $0.8 \mathrm{ml}$ sterile PBS. BAL fluid was centrifuged and single-cell suspensions were generated for staining. For analysis of lung tissue, mice were perfused with sterile PBS and the right inferior lung lobes were digested at $37^{\circ} \mathrm{C}$ with $630 \mu \mathrm{g} \mathrm{ml}^{-1}$ collagenase $\mathrm{D}$ (Roche) and $75 \mathrm{U} \mathrm{ml}^{-1}$ DNase I (Sigma-Aldrich) for $2 \mathrm{~h}$. Single-cell suspensions of BAL and lung digests were preincubated with Fc Block antibody (BD Pharmingen) in $\mathrm{PBS}+2 \%$ heat-inactivated FBS for $10 \mathrm{~min}$ at room temperature before staining. Cells were incubated with antibodies against the following markers: AF700 anti-CD45 (clone $30 \mathrm{~F}-11$ ), APC-Cy7 anti-CD11c (clone N418), PE anti-Siglec F (clone E50-2440; BD), PE-Cy7 anti-Ly6G (clone 1A8), BV605 anti-Ly6C (clone HK1.4; BioLegend), BV 711 anti-CD11b (clone M1/70), APC anti-CD103 (clone 2E7; eBioscience), $\mathrm{PB}$ anti-CD3 (clone 17A2), PE-Cy7, APC anti-CD4 (clone RM4-5), PE-Cy7 anti-CD8 (clone53-6.7), anti-NK1.1 (clone PK136) and BV605 anti-TCR $\gamma / \delta$ (clone GL3). All antibodies were used at a dilution of 1:200. Cells were stained for $20 \mathrm{~min}$ at $4{ }^{\circ} \mathrm{C}$, washed, fixed and permeabilized for intracellular staining with a Foxp3/Transcription Factor Staining Buffer Set (eBioscience) according to the manufacturer's instructions. Cells were incubated overnight at $4{ }^{\circ} \mathrm{C}$ with PE-Cy5 anti-Foxp3 (clone FJK-16s), washed, re-fixed with $4 \%$ paraformaldehyde (EMS) for $20 \mathrm{~min}$ and resuspended in permeabilization buffer. Absolute cell counts were determined using Trucount beads (BD). Flow cytometry data were acquired on a cytometer (BD X-20; BD Biosciences) and analyzed using FlowJo software (Treestar)

Flow cytometry analysis of peripheral blood. Peripheral blood was obtained from the submandibular vein. Erythrocytes were lysed twice with ammoniumchloride-potassium (ACK) lysing buffer (Gibco) and the remaining cells were resuspended in PBS supplemented with $2 \%$ FBS and $1 \mathrm{mM}$ EDTA and maintained on ice. After blockade of Fcy receptors with anti-CD16/32 (eBioscience; clone 93) and confirmation of viability (eBioscience; FVD eFluor 506), staining for the cell-surface antigens CD45 BUV395, CD3 APC, CD19 PE, Ly6G BV650, Ly6C Pacific Blue, CD11b PE/Dazzle 594, NK1.1 FITC, CD4 BV605 and CD8 PerCPCy5.5 was performed at dilutions of 1:200. Cells were incubated for $20 \mathrm{~min}$ at $4^{\circ} \mathrm{C}$, fixed with $4 \%$ paraformaldehyde for $20 \mathrm{~min}$ and washed before resuspension in PBS supplemented with 2\% FBS and 1 mM EDTA. Absolute cell counts were determined using Trucount beads (BD Biosciences). Flow cytometry data were acquired on a BD X-20 cytometer (BD Biosciences) and analyzed using FlowJo software (Treestar).

Clinical laboratory analysis. Testing was performed on fresh whole-blood samples within a biosafety cabinet using point-of-care instruments. Prothrombin time was measured using the CoaguChek (Roche) meter. Electrolyte, acid-base and hematology parameters were assayed on lithium-heparinized whole blood using the iSTAT 1 (Abbot) with the CHEM8+ cartridge.

Respiratory mechanics. Mice were anesthetized with ketamine/xylazine (100 and $10 \mathrm{mg} \mathrm{kg}^{-1}$ intraperitoneally, respectively). The trachea was isolated via dissection of the neck area and cannulated using an 18-gauge blunt metal cannula (typical resistance of $0.18 \mathrm{cmH}_{2} \mathrm{O} \mathrm{sml}^{-1}$ ), which was secured in place with a nylon suture. The mouse was then connected to the flexiVent computer-controlled piston ventilator (SCIREQ) via the cannula, which was attached to the FX adaptor Y-tubing. Mechanical ventilation was initiated and mice were given an additional $100 \mathrm{mg} \mathrm{kg}^{-1} \mathrm{ketamine}$ and $0.1 \mathrm{mg}$ per mouse of the paralytic pancuronium bromide via the intraperitoneal route to prevent breathing efforts against the ventilator and during measurements. Mice were ventilated using default settings for mice, 
which consisted of a positive end expiratory pressure at $3 \mathrm{cmH}_{2} \mathrm{O}$, a $10 \mathrm{ml} \mathrm{kg}^{-1}$ tidal volume, a respiratory rate at 150 breaths per minute and a fraction of inspired oxygen of 0.21 (that is, room air). Respiratory mechanics were assessed using the forced oscillation technique, as previously described ${ }^{49}$, using the latest version of the flexiVent operating software (flexiWare version 8.1.3). Pressure-volume loops and measurements of inspiratory capacity were also done.

Treadmill stress test. A six-lane mouse treadmill (Columbus Instruments) was placed within a biosafety cabinet within the animal biosafety level 3 laboratory. Mice were introduced to the treadmill test three times before infection, with each introductory session performed at increasingly faster rates. In general, the treadmill was set to ramp from 0 to maximum speed over the course of the first minute, then to maintain maximum speed for $5 \mathrm{~min}$. Failure to maintain adequate speed resulted in delivery of a shock; this occurred until the animal re-engaged the treadmill for a maximum of five failures. For each sex, we identified a speed at which $>80 \%$ of mice successfully completed the test before infection $\left(16 \mathrm{~m} \mathrm{~s}^{-1}\right.$ for females; $14 \mathrm{~m} \mathrm{~s}^{-1}$ for males).

RNA-seq. cDNA libraries were constructed starting with 10 ng total RNA from lung tissues of each sample that was extracted using a MagMAX mirVana Total RNA Isolation Kit (Thermo Fisher Scientific). cDNA was generated using the SeqPlex kit (Sigma-Aldrich) with amplification of 20 cycles. Library construction was performed using $100 \mathrm{ng}$ cDNA undergoing end repair, A tailing, ligation of universal TruSeq adapters and amplification of eight cycles to incorporate unique dual index sequences. Libraries were sequenced on the NovaSeq 6000 (Illumina) targeting 40 million read pairs and extending 150 cycles with paired-end reads. RNA-seq reads were aligned to the mouse Ensembl GRCh38.76 primary assembly and SARS-CoV-2 NCBI NC 045512 Wuhan-Hu-1 genome with the STAR program (version 2.5.1a $)^{50}$. Gene counts were derived from the number of uniquely aligned unambiguous reads by Subread:featureCount (version 1.4.6-p5) ${ }^{51}$. The ribosomal fraction, known junction saturation and read distribution over known gene models

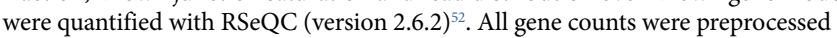
with the R package EdgeR $\mathrm{R}^{53}$ to adjust samples for differences in library size using the trimmed mean of $M$ values (TMM) normalization procedure. Ribosomal genes and genes not expressed at a level greater than or equal to 1 count per million reads in the smallest group size were excluded from further analysis. The R package limma ${ }^{54}$ with the voomWithQualityWeights function ${ }^{55}$ was utilized to calculate the weighted likelihoods for all samples, based on the observed mean-variance relationship of every gene and sample. Differentially expressed genes were defined as those with at least twofold difference between two individual groups at the Benjamini-Hochberg false discovery rate-adjusted $P$ value (that is, $q$ value $<0.05$ )

Statistical analysis. Statistical significance was assigned when $P$ values were $<0.05$ using Prism version 8 (GraphPad) and specific tests with exact $n$ values are indicated in the figure captions. Analysis of weight change was determined by two-way analysis of variance (ANOVA). Changes in functional parameters or immune parameters were compared with mock-infected animals and were analyzed by one-way ANOVA or one-way ANOVA with Dunnett's test.

Reporting Summary. Further information on research design is available in the Nature Research Reporting Summary linked to this article.

\section{Data availability}

All of the data supporting the findings of this study can be found within the paper and Extended Data Figs. 1-5 and are available from the corresponding author upon request or in the corresponding source data files. The RNA-seq datasets generated in this study are available from the Gene Expression Omnibus (accession code: GSE154104). The RNA-seq reads were aligned to the mouse Ensembl data (GRCh38.76 primary assembly) and GenBank data (SARS-CoV-2 NCBI NC_045512 Wuhan-Hu-1 genome). Source data are provided with this paper.

\section{References}

48. Mukherjee, S. et al. Enhancing dengue virus maturation using a stable furin over-expressing cell line. Virology 497, 33-40 (2016).
49. McGovern, T. K., Robichaud, A., Robichaud, L., Schuessler, T. F. \& Martin, J. G. Evaluation of respiratory system mechanics in mice using the forced oscillation technique. J. Vis. Exp. 2013, e50172 (2013).

50. Dobin, A. et al. STAR: ultrafast universal RNA-seq aligner. Bioinformatics $\mathbf{2 9}$, 15-21 (2013).

51. Liao, Y., Smyth, G. K. \& Shi, W. featureCounts: an efficient general purpose program for assigning sequence reads to genomic features. Bioinformatics 30, 923-930 (2014)

52. Wang, L., Wang, S. \& Li, W. RSeQC: quality control of RNA-seq experiments. Bioinformatics 28, 2184-2185 (2012).

53. Robinson, M. D., McCarthy, D. J. \& Smyth, G. K. edgeR: a Bioconductor package for differential expression analysis of digital gene expression data. Bioinformatics 26, 139-140 (2010).

54. Ritchie, M. E. et al. limma powers differential expression analyses for RNA-sequencing and microarray studies. Nucleic Acids Res. 43, e47 (2015).

55. Liu, R. et al. Why weight? Modelling sample and observational level variability improves power in RNA-seq analyses. Nucleic Acids Res. 43, e97 (2015).

\section{Acknowledgements}

This study was supported by NIH contracts and grants (75N93019C00062, R01 AI127828, R01 AI130591 and R35 HL145242) and the Defense Advanced Research Projects Agency (HR001117S0019). E.S.W. is supported by T32 AI007163. B.T.M. is supported by F32 AI138392. L.-I.K. is supported by T32 EB021955. We thank S. Whelan, S. Cook and J. Philips for facilitating the studies with SARS-CoV-2 at biosafety level 3; C Lutz and The Jackson Laboratory for providing mice; A. Kim for purifying the CR3022 anti-S monoclonal antibody; $\mathrm{H}$. Janova and M. Cain for experimental advice; and R. Schmidt for reviewing brain histology slides. We also thank D. Brunet at SCIREQ for facilitating use of the flexiVent mouse ventilator.

\section{Author contributions}

A.L.B. and E.S.W. performed the intranasal inoculations of SARS-CoV-2 and clinical analysis. E.S.W., J.M.F. and R.E.C. performed the viral burden analysis with support from J.T.E. N.M.K. performed the histopathological studies. B.T.M. performed the in situ hybridization. S.P.K., J.H.R., L.-I.K. and M.J.H. analyzed the tissue sections for histopathology. S.N. and E.S.W. performed immune cell processing for flow cytometry and analysis. A.L.B. performed the pulmonary mechanics analysis with training from S.D. S.D. and A.R. performed the analysis of pulmonary mechanics data. A.L.B. and N.M.K. performed the treadmill stress-testing analyses. J.Y. and R.H. performed the RNA-seq and analysis. E.S.W. compiled all of the figures. A.L.B., E.S.W. and M.S.D. wrote the initial draft, with the other authors providing editorial comments.

\section{Competing interests}

M.S.D. is a consultant for InBios, Vir Biotechnology and NGM Biopharmaceuticals and is on the scientific advisory board of Moderna. The Diamond laboratory has received funding under sponsored research agreements from Moderna, Vir Biotechnology and Emergent BioSolutions. S.D. and A.R. are employed by SCIREQ - a commercial entity with commercial interest in a subject area related to the content of this article. SCIREQ is an emka TECHNOLOGIES company. M.J.H. is a member of the Data and Safety Monitoring Board for AstroZeneca and founder of NuPeak Therapeutics. The other authors declare no competing interests.

\section{Additional information}

Extended data Extended data is available for this paper at https://doi.org/10.1038/ s41590-020-0778-2.

Supplementary information Supplementary information is available for this paper at https://doi.org/10.1038/s41590-020-0778-2.

Correspondence and requests for materials should be addressed to M.S.D

Peer review information Peer reviewer reports are available. Jamie D. K. Wilson was the primary editor on this article and managed its editorial process and peer review in collaboration with the rest of the editorial team.

Reprints and permissions information is available at www.nature.com/reprints. 
a

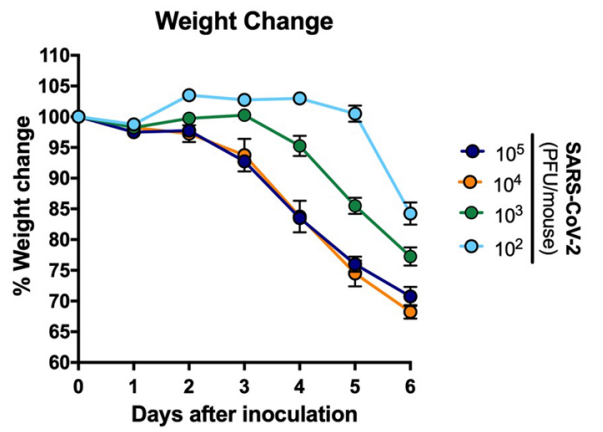

b

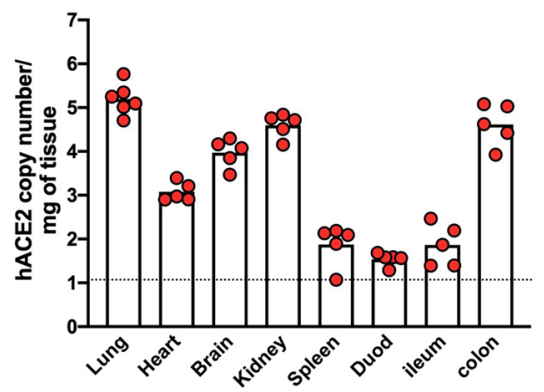

C

hACE2 Expression: Lung

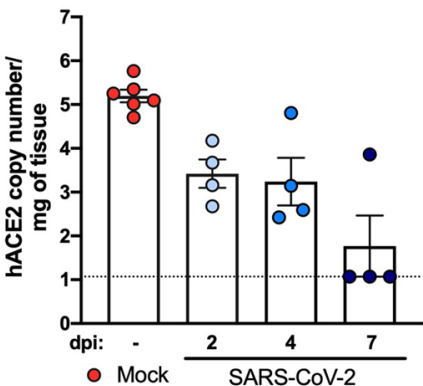

Extended Data Fig. 1 | SARS CoV-2 infection and hACE2 expression in the K18-hACE2 model. a. Six-week-old male and female K18-hACE2 transgenic mice were inoculated via the intranasal route with the indicated PFU doses of SARS-CoV-2, and weight change was monitored (one experiment, $n=4$, symbols represent the mean $\pm S E M)$. b. mRNA expression levels of hACE2 in the lung, kidney, heart, brain, spleen, duodenum, colon, and ileum of naive K18 hACE2 mice (two experiments, $n=5$ ). c. mRNA expression levels of hACE2 in the lungs of K18 hACE2 mice at 2, 4, and 7 dpi following SARS-CoV-2 infection (two experiments, $n=4$ per time point, bars represent the mean $\pm S E M$ ). 
a
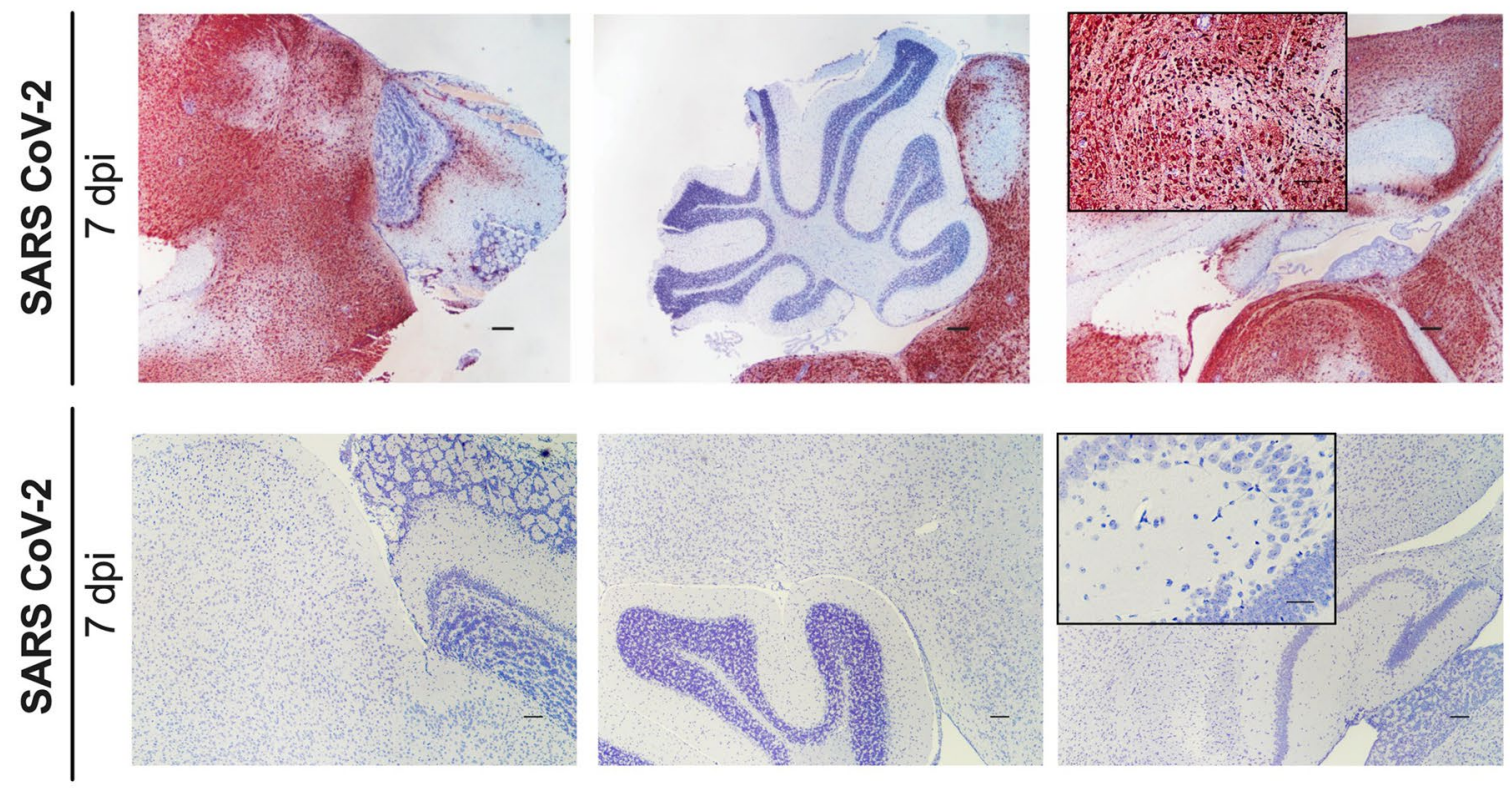

b
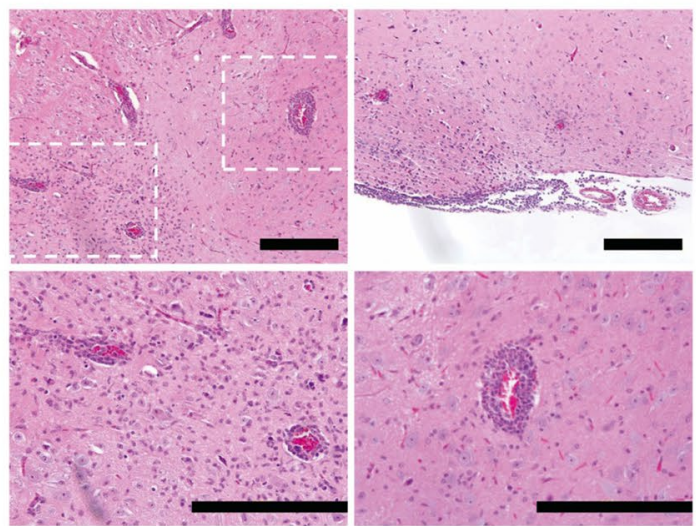

C

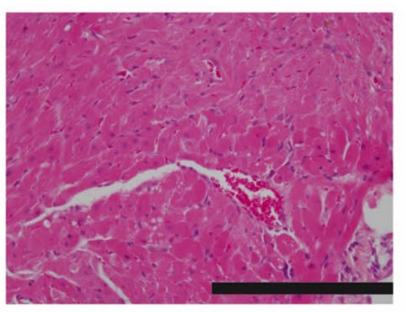

e

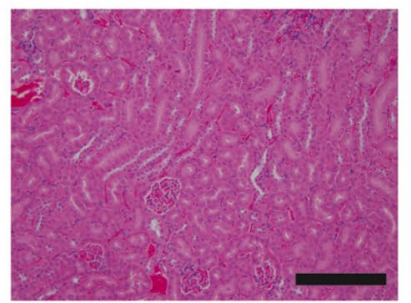

d

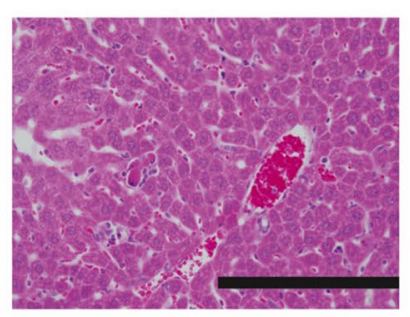

f

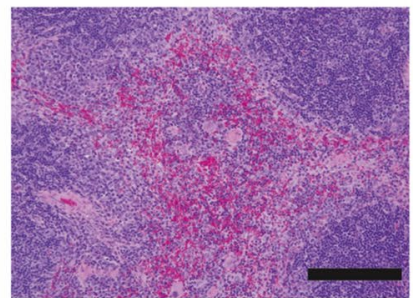

Extended Data Fig. 2 | SARS-CoV-2 infection in extra-pulmonary organs. a. SARS-CoV-2 RNA in situ hybridization of brain sections from K18-hACE2 mice following intranasal infection with $2.5 \times 10^{4}$ PFU of SARS-CoV-2 at 7 dpi. Images show low-power magnification (scale bars, $100 \mu \mathrm{m}$ ) with a high-power inset. One of six infected mice stained positively for viral RNA in the brain. Images are from this mouse and another that showed virtually no infection in the brain. b-e. Hematoxylin and eosin staining of the brain (b), heart (c), liver (d), kidney (e), and spleen (f) from K18-hACE2 mice following SARS CoV-2 infection at $7 \mathrm{dpi}$. Scale bars indicate $200 \mu \mathrm{m}$. For $\mathbf{b}$, hematoxylin and eosin-stained images correspond to the mouse with high levels of viral RNA by ISH and show inflamed vessels with extravasation of immune cells into the brain parenchyma, microglial activation, and subarachnoid inflammation with involvement of the underlying parenchyma. The dashed box in $\mathbf{b}$ indicates the location of two higher power magnification images below. For a-f, Representative images from 2 independent experiments, $n=6$ per group. 
a
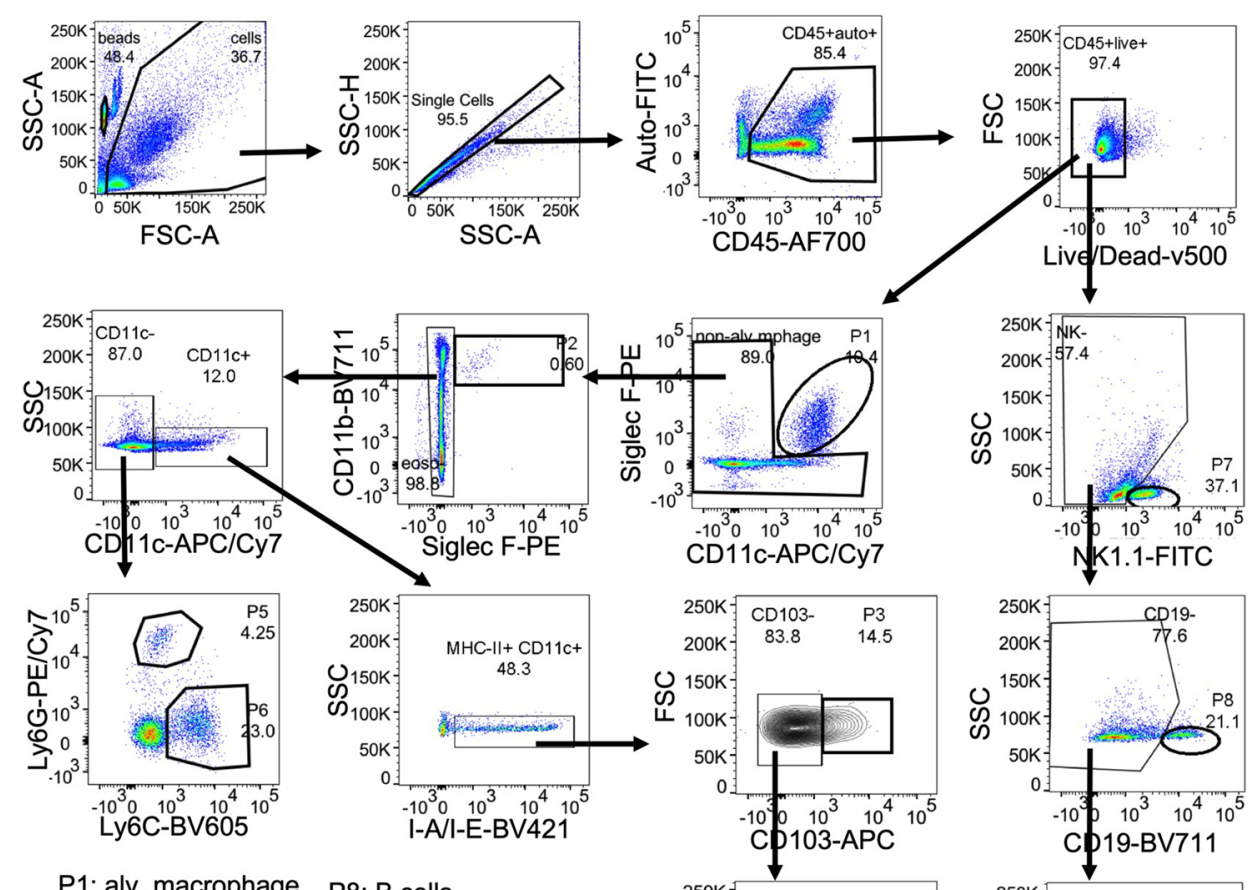

P1: alv. macrophage

P2: eosinophils

P3: $C D 103^{+} \mathrm{DCs}$

P4: CD11b+ DCs

P5: neutrophils

P8: B cells

P9: $\gamma \delta$ T cells

P10: CD4 cells

P11: CD8 cells

P12: Tregs

P6: inflammatory monocytes

P13: CD44 ${ }^{+}$CD8 cells

P7: NK cells
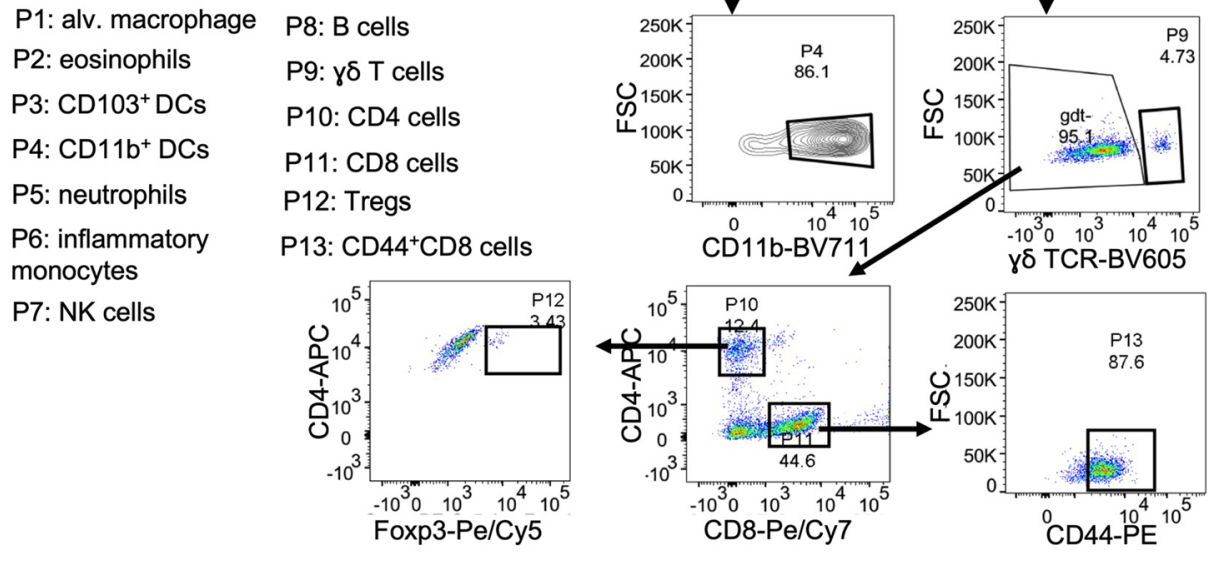

b
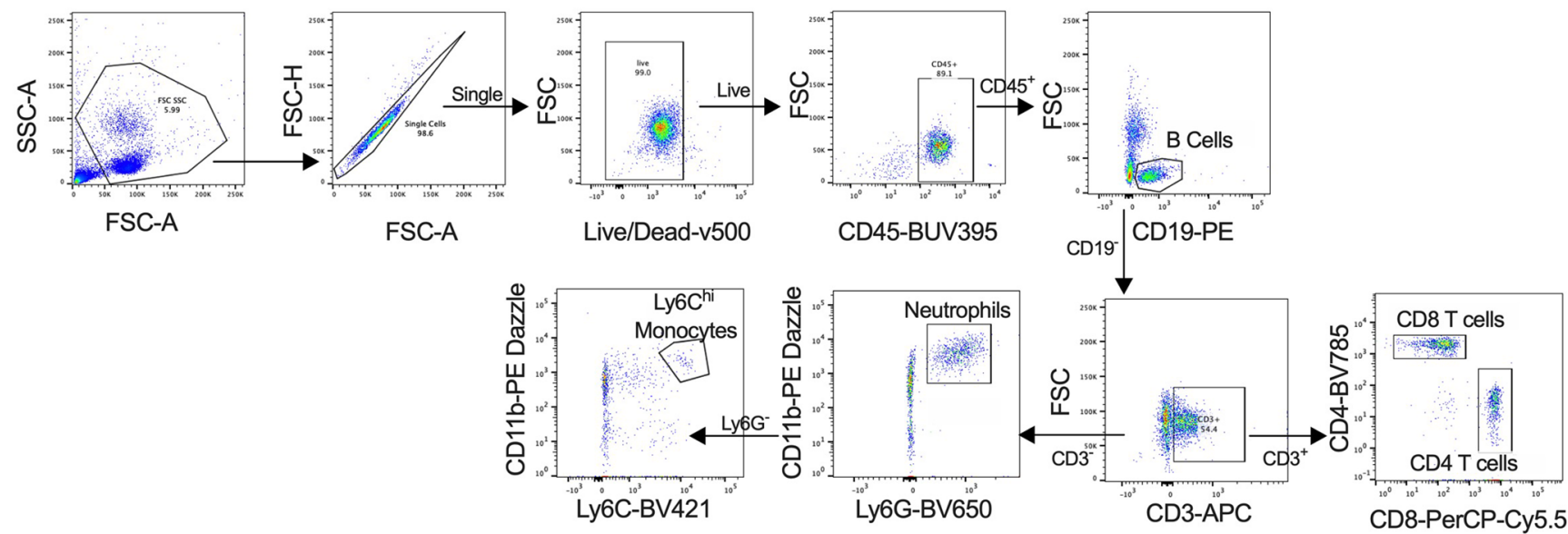

Extended Data Fig. 3 | Flow cytometric analysis. a. Flow cytometric gating strategy for BAL and lung tissue analysis. b. Flow cytometric gating strategy for peripheral blood leukocyte analysis. 

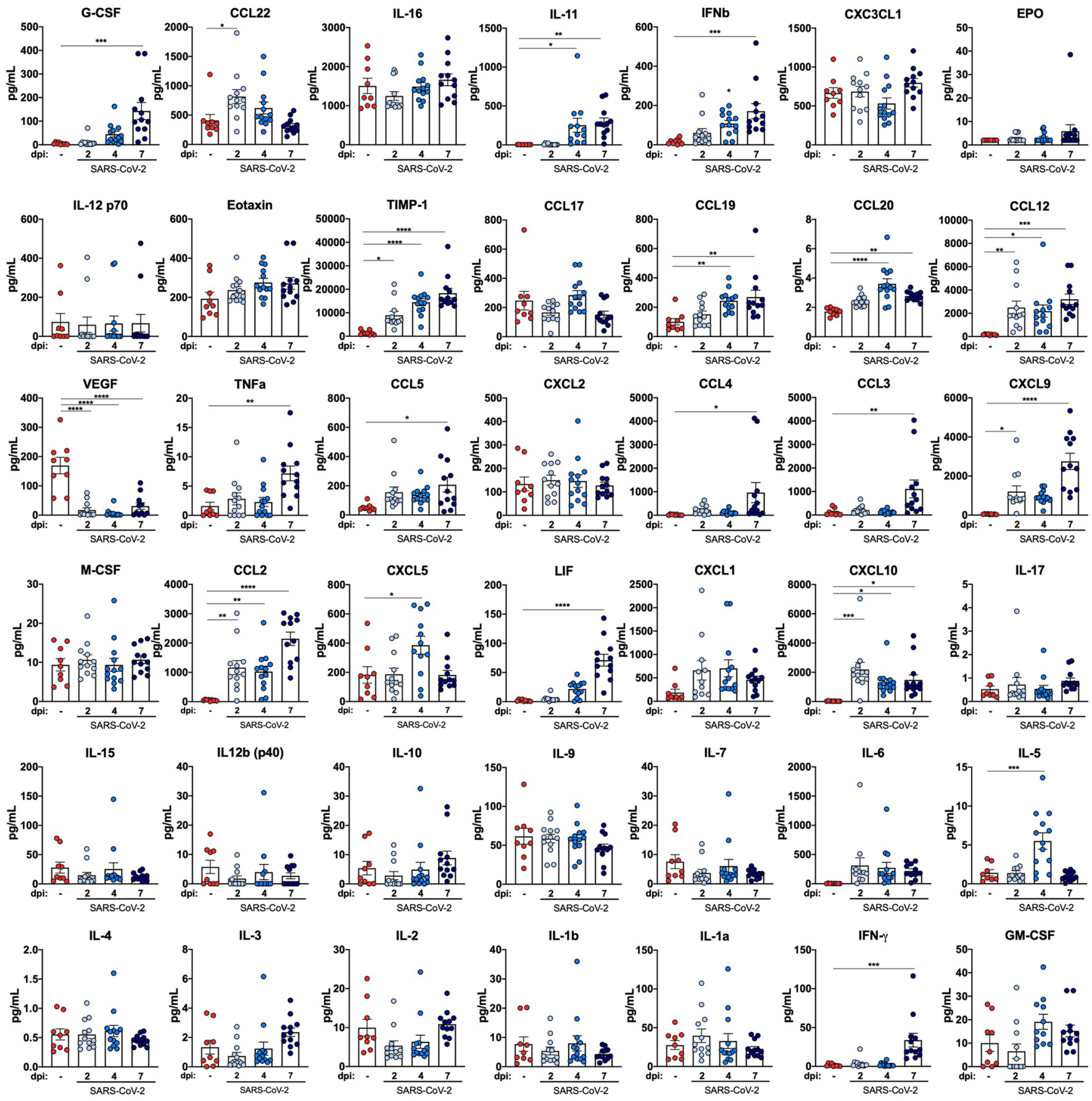

Extended Data Fig. 4 | Cytokine induction following SARS-CoV-2 infection. Cytokine levels as measured by multiplex platform in lung tissues of SARS-CoV-2-infected mice at 2, 4, and $7 \mathrm{dpi}$ (two independent experiments, $\mathrm{n}=9$ for naïve, $\mathrm{n}=12$ for $2 \mathrm{dpi}, \mathrm{n}=13$ for $4 \mathrm{dpi}, \mathrm{n}=12$ for $7 \mathrm{dpi}$; one-way ANOVA with Dunnett's test; for G-CSF ${ }^{\star \star \star} P=0.0001$; for $C C L 22{ }^{*} P=0.0181$; for IL-11 ${ }^{\star} P=0.0133$ and ${ }^{\star \star} P=0.0039$; for IFNb ${ }^{\star \star \star} P=0.0004$; for $\mathrm{TIMP}^{*}{ }^{\star} \mathrm{P}=0.0103$ and ${ }^{\star \star \star \star} \mathrm{P}<0.0001$; for CCL19 ${ }^{\star \star} \mathrm{P}=0.0076$; for CCL20 ${ }^{\star \star} \mathrm{P}=0.0053$ and ${ }^{\star \star \star \star} \mathrm{P}<0.0001$; for CCL12 ${ }^{\star} \mathrm{P}=0.0185$, ${ }^{\star \star} \mathrm{P}=0.0067$, and ${ }^{\star \star \star} \mathrm{P}=0.0004$; for VEGF ${ }^{\star \star \star \star} \mathrm{P}<0.0001$; for TNFa ${ }^{\star \star} \mathrm{P}=0.0015$; for $\mathrm{CCL}{ }^{\star}{ }^{\star} \mathrm{P}=0.0113$; for $\mathrm{CCL} 4{ }^{\star} \mathrm{P}=0.0203$; for $\mathrm{CCL3}{ }^{\star \star} \mathrm{P}=0.0054$; for $\mathrm{CXCL9}$ *

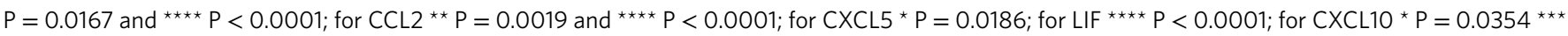
and $\mathrm{P}=0.0001$; for IL5 ${ }^{\star \star \star} \mathrm{P}=0.0005$; for IFNg ${ }^{\star \star \star} \mathrm{P}=0.0001$. Bars indicate the mean $\left.+/-\mathrm{SEM}\right)$ ). 
a

Neutrophil activation involved in immune response

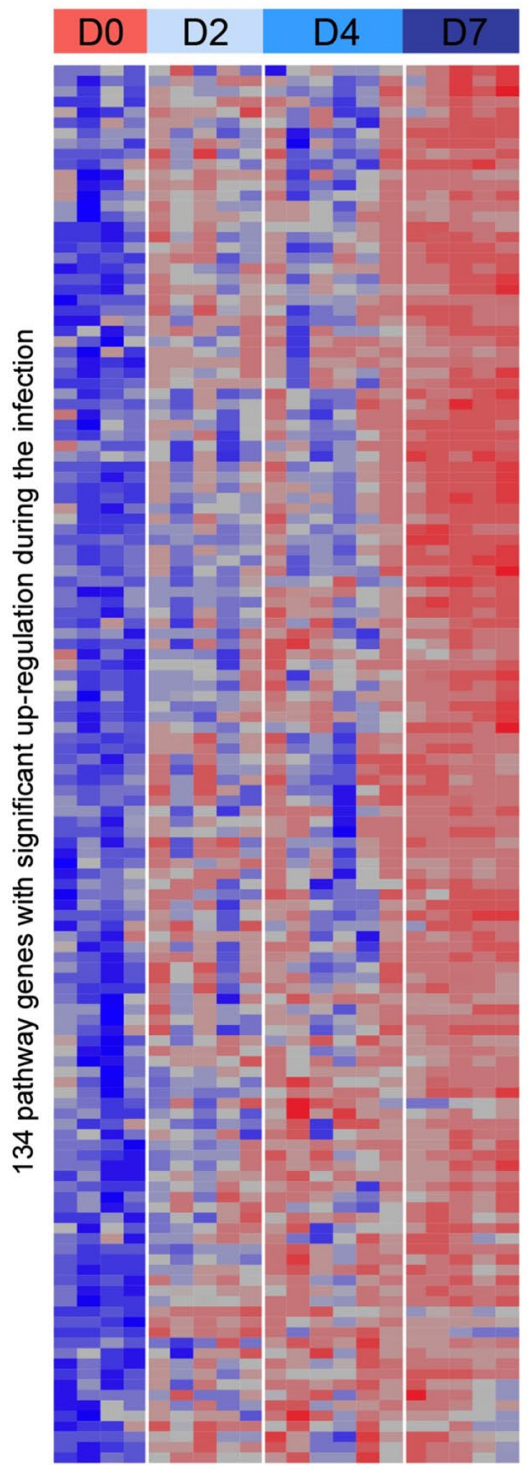

\section{b}

Toll-like receptor signaling pathway

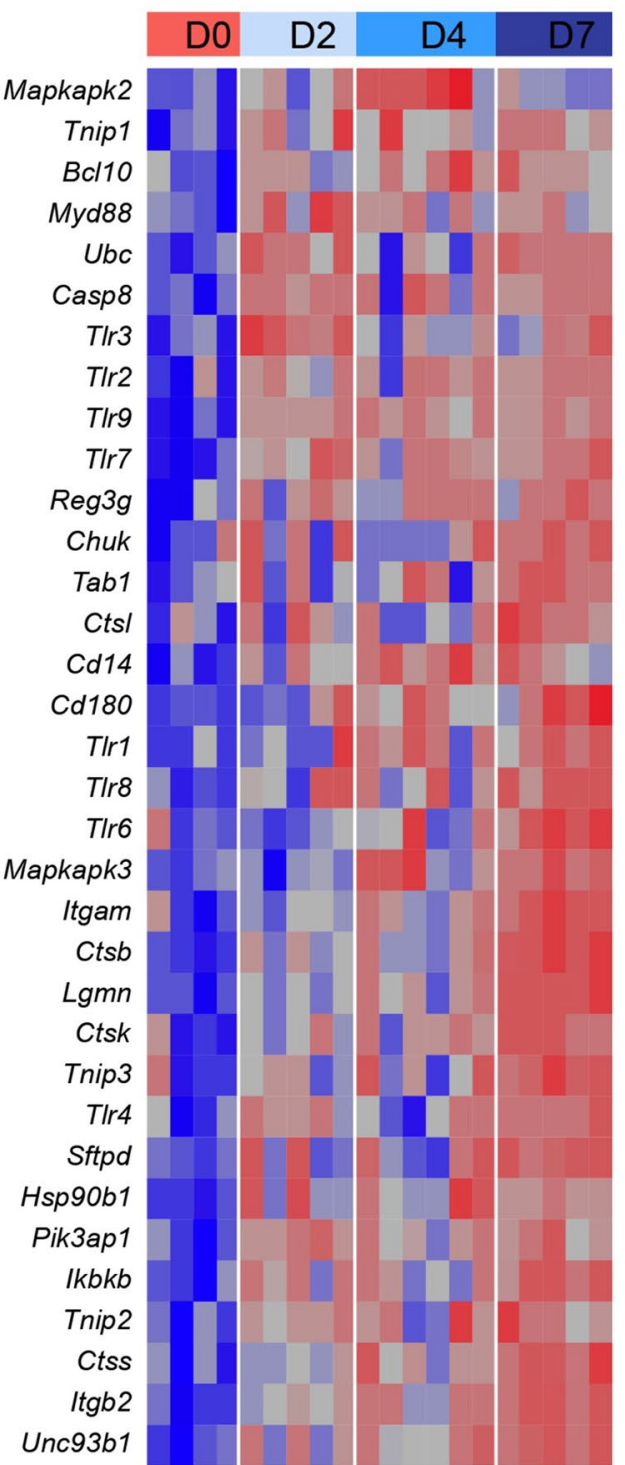

C

\section{Negative regulation of viral genome replication}

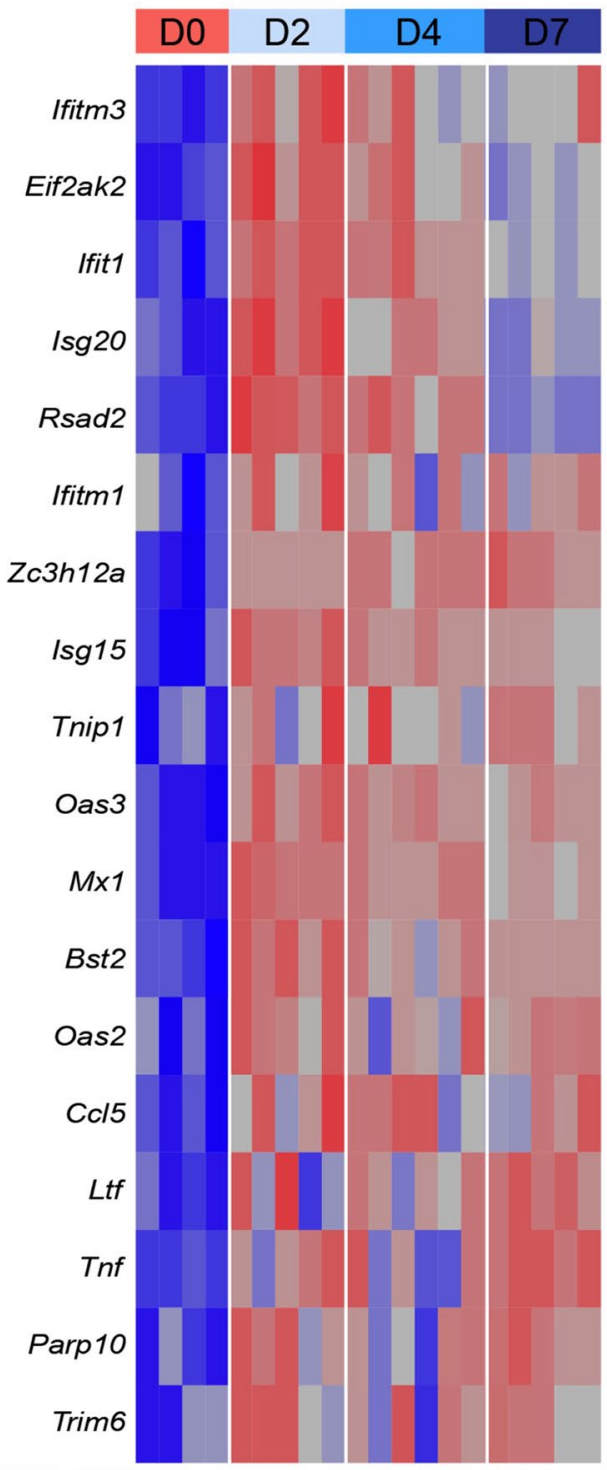

Low

Gene expression level

Extended Data Fig. 5 | Transcriptional immune signatures following SARS-CoV-2 infection. Heat maps of significantly upregulated genes during SARS-CoV-2 infection enriched in neutrophil activation pathways (a), Toll-like receptor signaling pathway (b), and negative regulation of viral genome replication (c) identified through Gene Ontology analysis. Genes shown in each pathway are the union of differentially expressed genes from the three comparisons $(2,4$, and 7 dpi versus mock-infected). Columns represent samples and rows represent genes. Gene expression levels in the heat maps are $z$ score-normalized values determined from log2 [cpm values]). 


\section{Reporting Summary}

Nature Research wishes to improve the reproducibility of the work that we publish. This form provides structure for consistency and transparency in reporting. For further information on Nature Research policies, see our Editorial Policies and the Editorial Policy Checklist.

\section{Statistics}

For all statistical analyses, confirm that the following items are present in the figure legend, table legend, main text, or Methods section.

$\mathrm{n} / \mathrm{a}$ Confirmed

$\bigotimes$ The exact sample size $(n)$ for each experimental group/condition, given as a discrete number and unit of measurement

\ A statement on whether measurements were taken from distinct samples or whether the same sample was measured repeatedly

The statistical test(s) used AND whether they are one- or two-sided

Only common tests should be described solely by name; describe more complex techniques in the Methods section.

\ A description of all covariates tested

$\square$ \ A description of any assumptions or corrections, such as tests of normality and adjustment for multiple comparisons

A full description of the statistical parameters including central tendency (e.g. means) or other basic estimates (e.g. regression coefficient)

AND variation (e.g. standard deviation) or associated estimates of uncertainty (e.g. confidence intervals)

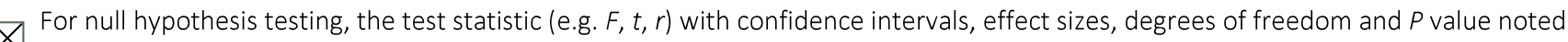

Give $P$ values as exact values whenever suitable.

Х $\square$ For Bayesian analysis, information on the choice of priors and Markov chain Monte Carlo settings

$\square$ \ For hierarchical and complex designs, identification of the appropriate level for tests and full reporting of outcomes

$\triangle \square$ Estimates of effect sizes (e.g. Cohen's d, Pearson's $r$ ), indicating how they were calculated

\section{Our web collection on statistics for biologists contains articles on many of the points above.}

\section{Software and code}

Policy information about availability of computer code

Data collection

CDNA libraries were constructed starting with $10 \mathrm{ng}$ of total RNA from lung tissues of each sample that was extracted using a MagMax mirVana Total RNA isolation kit (Thermo Scientific). cDNA was generated using the Seqplex kit (Sigma-Aldrich) with amplification of 20 cycles. Library construction was performed using $100 \mathrm{ng}$ of cDNA undergoing end repair, A tailing, ligation of universal TruSeq adapters and amplification of 8 cycles to incorporate unique dual index sequences. Libraries were sequenced on the NovaSeq 6000 (Illumina, San Diego, CA) targeting 40 million read pairs and extending 150 cycles with paired end reads.

Flow cytometry data were acquired on a BD-X20 cytometer (BD Biosciences) .

Respiratory mechanics data was acquired using a flexiVent computer-controlled piston ventilator (SCIREQ Inc.).

Data analysis

RNA-seq reads were aligned to the mouse Ensembl data (GRCh38.76 primary assembly) and Genbank data (SARS-CoV-2 NCBI NC_045512 Wuhan-Hu-1 genome) with STAR program (version 2.5.1a). Gene counts were derived from the number of uniquely aligned unambiguous reads by Subread:featureCount (version 1.4.6-p5). The ribosomal fraction, known junction saturation, and read distribution over known gene models were quantified with RSeQC (version 2.6.2). All gene counts were preprocessed with the R package EdgeR v4.0.1 to adjust samples for differences in library size using the trimmed mean of $\mathrm{M}$ values (TMM) normalization procedure. Ribosomal genes and genes not expressed at a level greater than or equal to 1 count per million reads in the smallest group size were excluded from further analysis. The R package limma with voomWithQualityWeights v4.0.1 function was utilized to calculate the weighted likelihoods for all samples, based on the observed mean-variance relationship of every gene and sample. Differentially expressed genes were defined as those with at least 2-fold difference between two individual groups at the Benjamini-Hochberg false-discovery rate (FDR) adjusted p-value, i.e. q-value $<0.05$.

This study used commercially available Graphpad prism software v8.2.1 for data representation and statistical analysis. FlowJo v 10.5.3 was used to analyze all flow cytometry data. Flexiware v8.1, service pack 3 was used to analyze Flexivent data. 
Policy information about availability of data

All manuscripts must include a data availability statement. This statement should provide the following information, where applicable:

- Accession codes, unique identifiers, or web links for publicly available datasets

- A list of figures that have associated raw data

- A description of any restrictions on data availability

All data supporting the findings of this study are found within the paper and its Extended Data Figures, and are available from the corresponding author upon request. RNA sequencing data sets generated in this study are available at GEO: GSE154104.

\section{Field-specific reporting}

Please select the one below that is the best fit for your research. If you are not sure, read the appropriate sections before making your selection.

\ Life sciences

Behavioural \& social sciences

Ecological, evolutionary \& environmental sciences

For a reference copy of the document with all sections, see nature.com/documents/nr-reporting-summary-flat.pdf

\section{Life sciences study design}

All studies must disclose on these points even when the disclosure is negative.

Sample size

No sample-size calculations were performed to power each study. Sample sizes for mouse studies were determined based on previous results for similar in vivo experiments which showed that the use of 5-12 mice per group represents a minimally sufficient sample to produce a study power of $>80 \%$ (PMID: 32553273 ).

No experiments not utilizing mice or downstream analysis of samples collected from mice was included in this study.

Data exclusions One mouse was excluded from donwstream analysis due to an injury during handling. This mouse was excluded from all downstream analysis (cytokine analysis, blood chemistry analysis, flexivent analysis, RNAseq analysis). As the injury occured at 6 dpi, weight loss data and treadmill data from this mouse that occurred prior to the injury was still included in final analysis in there two parameters.

Replication All experiments were performed in at least 2 independent biological repeats. All attempts at replication were successful.

Randomization Mice were randomly assigned to each treatment group (mock, $2 \mathrm{dpi}$, 4, dpi and $7 \mathrm{dpi}$ ).

No experiments not utilizing mice or downstream analysis of the mice was included in this study.

Blinding Investigators were not blinded for the following analyses (clinical measurements, viral burden analysis and immunological analysis) due to the biosafety concerns associated with handling these samples. For histological analyses slides were blinded prior to scoring. For RNAseq data, investigators were unblinded as to sample identities only after initial analysis. Investigators were unblinded after initial analysis in order to dissect biological pathways in the context of the other data included in this paper that could be mechanistically informative in the RNAseq data.

\section{Reporting for specific materials, systems and methods}

We require information from authors about some types of materials, experimental systems and methods used in many studies. Here, indicate whether each material, system or method listed is relevant to your study. If you are not sure if a list item applies to your research, read the appropriate section before selecting a response.

\begin{tabular}{l|l} 
Materials \& experimental systems \\
\hline $\mathrm{n} / \mathrm{a}$ & Involved in the study \\
$\square$ & $\bigotimes$ Antibodies \\
$\square$ Eukaryotic cell lines \\
$\square$ Palaeontology and archaeology \\
$\square$ & $\square$ Animals and other organisms \\
$\square$ & $\square$ Human research participants \\
$\square$ & $\square$ Clinical data
\end{tabular}

\begin{tabular}{l|l}
\multicolumn{2}{l}{ Methods } \\
\hline n/a & Involved in the study \\
$\square$ & $\square$ ChIP-seq \\
$\square$ & $\square$ Flow cytometry \\
$\square$ & $\square$ MRI-based neuroimaging
\end{tabular}


AF700 anti-CD45 (Biolegend, Cat \#103127, clone 30 F-11), APC-Cy7 anti-CD11c (Biolegend, Cat \#117323, clone N418), PE anti-Siglec F (BD, Cat \#552126, clone E50-2440), PE-Cy7 anti-Ly6G (Biolegend, Cat \# 127607, clone 1A8), BV605 anti-Ly6C (Biolegend, Cat \# 128035, clone HK1.4) BV 711 anti-CD11b (Biolegend, Cat \#101241, clone M1/70), APC anti-CD103 (eBioscience, Cat\# 17-1031-82, clone 2E7), Pacific Blue anti-CD3 (Biolegend, Cat \# 100213, clone 17A2), APC anti-CD4 (Biolegend, Cat \# 100515, clone RM4-5), PECy7 anti-CD8 (Biolegend, Cat \# 100721, clone53-6.7), FITX anti-NK1.1 (Biolegend, Cat \#108705 clone PK136), BV605 anti-TCR y/8 (Biolegend, Cat \# 118129, clone GL3), PE-Cy5 anti-Foxp3 (eBioscience, Cat\# 15-5773-82, clone FJK-16s), Fc Block (Biolegend, Cat\#101301, clone 93), BUV395 CD45 (BD, Cat \#564279 clone Clone 30-F11), APC anti-mouse CD3 Antibody (Biolegend, Cat\# 100235, Clone 17A2), PE anti-mouse CD19 Antibody (Biolegend, Cat\#152407, Clone 1D3), Brilliant Violet 650 ${ }^{\text {TM }}$ anti-mouse Ly-6G Antibody (Biolegend, Cat \#127641, Clone 1A8), Pacific Blue ${ }^{\mathrm{TM}}$ anti-mouse Ly-6C Antibody (Biolegend, Cat \#128013, Clone HK1.4), PE/ Dazzle ${ }^{\text {TM }} 594$ anti-mouse/human CD11b Antibody (Biolegend, Cat\# 101255, Clone, Brilliant Violet 605 ${ }^{\text {TM }}$, anti-mouse/human CD11b Antibody (Biolegend, Cat \#101237, Clone M1/70), PerCP/Cyanine5.5 anti-mouse CD8a Antibody (Biolegend., Cat \# 100733, Clone 53-6.7), Fixable Viability Dye eFluor ${ }^{\mathrm{TM}} 506$ ( $^{\text {eBioscience }}{ }^{\mathrm{TM}}$, Cat \#65-0866-14)

Validation

The antibody panels has been used previously for the same application (PMID 29511063, PMID: 32668198).

For primary antibody validation product citations on the the manufacturers website are listed below.

1. AF700 anti-CD45 (Biolegend, Cat \#103127, clone 30 F-11): PMID: 22611244, PMID: 22844125, PMID: 25404286

2. APC-Cy7 anti-CD11c (Biolegend, Cat \#117323, clone N418): PMID: 23065153

3. PE anti-Siglec F (BD, Cat \#552126, clone E50-2440): PMID: 11579105

4. PE-Cy7 anti-Ly6G (Biolegend, Cat \# 127607, clone 1A8): PMID: 22474024

5. BV605 anti-Ly6C (Biolegend, Cat \# 128035, clone HK1.4) : PMID: 30318149

6. BV 711 anti-CD11b (Biolegend, Cat \#101241: PMID: 25964477

7. APC anti-CD103 (eBioscience, Cat\# 17-1031-82, clone 2E7: PMID: 28533230

8. Pacific Blue anti-CD3 (Biolegend, Cat \# 100213, clone 17A2): PMID: 19234143

9. PE-Cy7 anti-CD8 (Biolegend, Cat \# 100721, clone53-6.7): PMID: 22952867

10. APC anti-CD4 (Biolegend, Cat \# 100515, clone RM4-5): PMID: 17277158

11. FITC anti-NK1.1 FITC (Biolegend, Cat \#108705 clone PK136),PMID: 25155355

12. BV605 anti-TCR $\gamma / \delta$ (Biolegend, Cat \# 118129, clone GL3), PMID: 26587585

13. PE-Cy5 anti-Foxp3 (eBioscience, Cat\# 15-5773-82, clone FJK-16s)PMID: 30410056

14. Fc Block (Biolegend, Cat\#101301, clone 93) : PMID: 15699180

15. BUV395 CD45 (BD, Cat \#564279 clone Clone 30-F11) : PMID: 11062533

16. APC anti-mouse CD3 Antibody (Biolegend, Cat\# 100235, Clone 17A2): PMID: 26169940

17. PE anti-mouse CD19 Antibody (Biolegend, Cat\#152407, Clone 1D3): PMID: 30127434

18. Brilliant Violet $650^{\mathrm{TM}}$ anti-mouse Ly-6G Antibody (Biolegend, Cat \#127641, Clone 1A8): PMID: 27731313

19. Pacific Blue ${ }^{T M}$ anti-mouse Ly-6C Antibody (Biolegend, Cat \#128013, Clone HK1.4): PMID: 22043017

20. PE/Dazzle ${ }^{\text {TM }} 594$ anti-mouse/human CD11b Antibody (Biolegend, Cat\# 101255, Clone M1/70): PMID: 26604307

21. anti-mouse/human CD11b Antibody (Biolegend, Cat \#101237, Clone M1/70): PMID: 24043758

22. PerCP/Cyanine5.5 anti-mouse CD8a Antibody (Biolegend., Cat \# 100733, Clone 53-6.7): PMID: 23460738

23. Fixable Viability Dye eFluor ${ }^{\text {TM }} 506$ ( eBioscience ${ }^{\text {TM }}$, Cat \#65-0866-14): PMID: 32668198

\section{Eukaryotic cell lines}

Policy information about cell lines

Cell line source(s)

In this study we used the following cell lines: Vero E6 (ATCC, Cat\# CRL-1586), Vero CCL81 (ATCC Cat\#CCL81) and Vero-Furin cells (Gift of T. Pierson, NIH; originally described in PMID: 27420797)

Authentication

Morphology for each cell line was assessed by microscope. Permissiveness of each cell line was assessed through observation of CPE by microscope and through quantification of virus produced by plaque assay, focus forming assay, and qPCR for viral RNA.

Mycoplasma contamination

All cells are tested on a monthly basis.

Commonly misidentified lines

(See ICLAC register)

None

\section{Animals and other organisms}

Policy information about studies involving animals; ARRIVE guidelines recommended for reporting animal research

Laboratory animals

In this study, the following mouse strain was used B6.Cg-Tg(K18-ACE2)2PrImn/J (https://www.jax.org/strain/034860). For viral challenge male and female eight-week old mice were challenged intranasally with SARS-CoV-2.

Mice were housed in groups of up to 5 mice/cage at 18 degrees C-24 degrees $C$ ambient temperatures with 40-60\% humidity. Mice were fed a 20\% protein diet (PicoLab 5053, Purina) and maintained on a 12 hour light/ dark cycle 6 am to 6 pm. Food and water were available ad libitum. 
Ethics oversight

Animal studies were carried out in accordance with the recommendations in the Guide for the Care and Use of Laboratory Animals of the National Institutes of Health. The protocols were approved by the Institutional Animal Care and Use Committee at the Washington University School of Medicine (assurance number A3381-01). Virus inoculations were performed under anesthesia that was induced and maintained with ketamine hydrochloride and xylazine, and all efforts were made to minimize animal suffering.

Note that full information on the approval of the study protocol must also be provided in the manuscript.

\section{Flow Cytometry}

\section{Plots}

Confirm that:

\The axis labels state the marker and fluorochrome used (e.g. CD4-FITC).

\The axis scales are clearly visible. Include numbers along axes only for bottom left plot of group (a 'group' is an analysis of identical markers).

$\bigotimes$ All plots are contour plots with outliers or pseudocolor plots.

\A numerical value for number of cells or percentage (with statistics) is provided.

\section{Methodology}

Sample preparation

Instrument

Software

Cell population abundance

Gating strategy
Single cell suspensions of BAL and lung digests were preincubated with Fc Block antibody (BD PharMingen) in PBS $+2 \%$ heatinactivated FBS for $10 \mathrm{~min}$ at room temperature before staining. Cells were incubated with antibodies against the following markers: AF700 anti-CD45 (clone 30 F-11), APC-Cy7 anti-CD11c (clone N418), PE anti-Siglec F (clone E50-2440; BD), PE-Cy7 anti-Ly6G (clone 1A8), BV605 anti-Ly6C (clone HK1.4; Biolegend), BV 711 anti-CD11b (clone M1/70), APC anti-CD103 (clone 2E7; eBioscience), PB anti-CD3 (clone 17A2), PE-Cy7, APC anti-CD4 (clone RM4-5), PE-Cy7 anti-CD8 (clone53-6.7), anti-NK1.1 (clone PK136), and BV605 anti-TCR $\gamma / \delta$ (clone GL3). All antibodies were used at a dilution of 1:200. Cells were stained for 20 min at $4^{\circ} \mathrm{C}$, washed, fixed and permeabilized for intracellular staining with Foxp3/Transcription Factor Staining Buffer Set (eBioscience) according to manufacturer's instructions. Cells were incubated overnight at $4^{\circ} \mathrm{C}$ with PE-Cy5 anti-Foxp3 (clone FJK-16s), washed, re-fixed with 4\% PFA (EMS) for $20 \mathrm{~min}$ and resuspended in permeabilization buffer.

For analysis of peripheral blood leukocytes, peripheral blood was obtained from the submandibular vein. Erythrocytes were lysed twice with ammonium chloride-potassium (ACK) lysing buffer (Gibco), and remaining cells were resuspended in PBS supplemented with 2\% FBS and $1 \mathrm{mM}$ EDTA and maintained on ice. After blockade of Fcy receptors with anti-CD16/32 (eBioscience; clone 93) and confirmation of viability (eBioscience; FVD efluor 506), staining for cell surface antigens CD45 BUV395, CD3 APC, CD19 PE, Ly6G BV650, Ly6C Pacific Blue, CD11b PE/Dazzle 594, NK1.1 FITC, CD4 BV605, and CD8 PerCPCy5.5 was performed at dilutions of 1:200. Cells were incubated for $20 \mathrm{~min}$ at $4^{\circ} \mathrm{C}$, fixed with $4 \%$ PFA for $20 \mathrm{~min}$, and washed prior to resuspension in PBS supplemented with $2 \%$ FBS and 1 mM EDTA. Absolute cell counts were determined using TruCount beads (BD Biosciences). Flow cytometry data were acquired on a BD-X20 cytometer (BD Biosciences) and analyzed using FlowJo software (Tree Star).

Flow cytometry data were acquired on a BD-X20; BD Biosciences

FlowJo v10.5.3

N/A. No cell sorting was performed in this study.

All gating strategies are shown in Extended Data Figures.

For lung tissues and BAL to myeloid cells:

FSC SSC to remove debris

SSC-H by SSC-A to define single cells

Autofluorescent by CD45 to identify hematopeitic cells

FSC by Live/Dead to identify live cells

SiglecF by CD11c to define alveolar macrophages

For not alveolar macrophages, CD11b+SigF+ were identified as eosiniphils

For not eosinophils, SSC by CD11c to define CD11c+ and CD11c- cells

For CD11c- cells, Ly6G by Ly6C to define neutrophils and monocytes respectively

For CD11c+ cells, SSC by MHCll+ to define dendritic cells

SSC by CD103+ to define, CD103+ DCs

For CD103-, CD11b+ to define CD11b+DCs

For lung tissues and BAL to lymphoid cells:

FSC SSC to remove debris

SSC-H by SSC-A to define single cells

Autofluorescent by CD45 to identify hematopeitic cells

FSC by Live/Dead to identify live cells

SSC by NK1.1 to define NK+ cells 
SSC by CD19+ to define B cells

FSC by gd TCR to define gamma-delta T cells

For gdTCR- cells, CD4 by CD8 to define CD4+ and CD8+ T cells

For CD4+ T cells, Fox3P to define Tregs

For CD8+ T cells, CD44 to define activated CD44+ T cells

For Peripheral blood:

FSC SSC to remove debris

FSC-H by FSC-A to define single cells

FSC by Live/Dead to identify live cells

FSC by CD45 to identify hematopoeitic cells

FSC by CD19+ to define B cells

For CD19-, FSC by CD3+ to define T cells

For CD3+, CD4 by CD8 to define CD4+ and CD8+ T cells

For CD3-, CD11b by Ly6G to define neutrophils

For Ly6G-, CD11b by Ly6C to define monocytes

$\bigotimes$ Tick this box to confirm that a figure exemplifying the gating strategy is provided in the Supplementary Information. 